\title{
Reconstruction of the envelope of non-Gaussian structural responses with principal static wind loads
}

\author{
Nicolas Blaise $^{\mathrm{a}, *}$, Thomas Canor ${ }^{\mathrm{b}}$, Vincent Denoël ${ }^{\mathrm{a}}$ \\ ${ }^{a}$ Department of Architecture, Geology, Environment and Construction, University of Liège, Liège, Belgium \\ ${ }^{b}$ F.R.S.-FNRS, National Fund for Scientific Research, University of Liège, Liège, Belgium
}

\begin{abstract}
In current practice, structural engineers commonly focus on the wind-resistant design by means of static wind loads. In case of non-Gaussianities, there is room for improvement to properly derive these static loads. First, this paper extends in a non-Gaussian context the concept of the loadresponse correlation (LRC) method establishing equivalent static wind loads (ESWLs). This is done by a proper recourse to the new concept of conditional expected static wind load and a proposed bicubic model for the joint and conditional distribution functions. Second, this paper investigates the envelope reconstruction problem targeting the efficient reconstruction of the envelope values of a set of non-Gaussian structural responses by means of principal static wind loads (PSWLs). They have been introduced in a Gaussian context and are obtained by a singular value decomposition of ESWLs. This paper addresses the extension of PSWLs to non-Gaussian structural responses, as well. The developments apply to structures with a linear behaviour and subjected to an aerodynamic pressure field exhibiting mildly to strongly non-Gaussian features. In this context, the well-known load-response correlation and conditional sampling methods are used for comparisons. This study is undertaken for quasi-static analysis of structures and is illustrated on a low-rise building.
\end{abstract}

Keywords: Non-Gaussian buffeting analysis; Extreme value; Equivalent static wind loads; Conditional expected static wind loads; Envelope reconstruction problem; Bicubic model

\section{Introduction}

The design of civil structures subjected to aerodynamic pressure fields by means of static wind loads is usual. Mainly, such an analysis should provide structural responses similar to the extreme values that would be provided by a buffeting analysis. Actually, these extreme values define an envelope and the structural analysis through static loads may be understood as an envelope reconstruction problem (Blaise and Denoël, 2013). This problem consists in the efficient reconstruction of extreme values of structural responses through a set of static loads. When they are known, they are readily applied to the structure in a straightforward analysis without repeating cumbersome dynamical analyses.

\footnotetext{
${ }^{*}$ Tel.: +32 4366 9208; fax:+32 43669192 .

Email addresses: n.blaise0@gmail.com (Nicolas Blaise), thomas.canor@gmail.com (Thomas Canor), v.denoel@ulg.ac.be (Vincent Denoël)
} 
Several methods have been developed to tackle the envelope reconstruction problem (e.g., Repetto and Solari (2004); Katsumura et al. (2007); Fiore and Monaco (2009); Zhou et al. (2011)), see (Blaise and Denoël, 2013) for a review. In these methods, several responses may be specified, overcoming the main limitation of the equivalent static wind loads (ESWLs) initially derived for one specific structural response. Indeed, an efficient solution of the envelope reconstruction problem requires to derive a limited number of static wind loads, each of them aiming at a global reconstruction; especially for complex structures like stadium roofs (Blaise et al., 2012). With this objective, Blaise and Denoël (2013) developed principal static wind loads (PSWLs) in a Gaussian context. These PSWLs result from the singular value decomposition to the matrix gathering ESWLs computed for all structural responses of interest.

The present paper aims at solving the envelope reconstruction problem with PSWLs in the case of non-Gaussian structural responses resulting from non-Gaussian aerodynamic pressures. A first contribution of this paper is the formulation of "non-Gaussian" ESWLs through the novel concept of conditional expected static wind load. This is discussed for structures with a quasi-static behavior and subjected to a mildly to strongly non-Gaussian aerodynamic pressure field. A second contribution is the generalization of PSWLs for non-Gaussian responses.

The conditional sampling technique and the load-response correlation (LRC) method are considered in this study for comparisons.

Holmes (1988) introduced the notion of peak-load pressure distributions. To each extreme response corresponds specific wind load patterns obtained with wind-tunnel tests. The conditional sampling technique (Atta, 1974) identifies these peak-load pressure distributions. This technique has been mainly applied to low-rise buildings (Tamura et al., 1992, 2001). Following the same idea of a load associated with a maximum response, Kasperski (1992) established the LRC method giving an ESWL corresponding to an envelope value obtained by a linear quasi-static analysis in a Gaussian framework.

The conditional sampling technique, by nature, incorporates the non-Gaussian aspects in both aerodynamic loads and structural responses. Conversely, the original gust loading factor and LRC methods and its extensions (Chen and Kareem, 2001) were developed assuming Gaussian processes. Actually, Kasperski (1992) sidesteps the extension to non-Gaussian processes arguing that, the LRC method would provide "[...] a very close approximation to the real load pattern [...]" even if the aerodynamic pressure field was non-Gaussian or if the structure had a weakly nonlinear behavior. However, differences have been shown between the LRC-based ESWLs and those obtained with statistical treatment of wind-tunnel measurements (Tamura et al., 2002). This has especially motivated the study of a non-Gaussian formulation for ESWLs.

The organization of this paper is as follows. First, non-Gaussian structural analysis and establishment of the asymmetrical envelope of structural responses are exposed (Section 2). Then the concept of conditional expected static wind load is introduced and a non-Gaussian formulation of ESWLs is derived by means of a bicubic model for the load-response joint distribution fonction (Section 3). Generalization of the PSWLs for the reconstruction of asymmetric envelopes and how to combine them to speed-up the envelope reconstruction problem are then described (Section 4). All the developments are illustrated with the non-Gaussian quasi-static analysis of a low-rise gable-roof building (Section $5)$. 


\section{Nomenclature}

Bold lowercase letters are used to denote vectors while bold uppercase is preferred for matrices. The $i$-th entry of $\mathbf{r}$ is denoted by $\mathrm{r}_{i}$ and the $(i, j)$-th entry of $\mathbf{R}$ is denoted by $\mathrm{R}_{i j}$. The $i$-th column of $\mathbf{R}$ is denoted by $\mathbf{R}_{i}$. While subscripts without bracket are used to specify entries in a vector or matrix, superscripts are always in bracket and are used to give additional information. Subscripts in bracket are only used in Sections 4.2 and 4.3 and denotes the number of the iteration considered in the envelope reconstruction problem.

We use the prime symbol ' to indicate a stochastic process with a non-zero mean, while the zeromean fluctuation is devoid of this symbol. Symbols $\boldsymbol{\mu}$. and $\boldsymbol{\sigma}$. are used to denote mean and standard deviation of vector entries. For instance, we write

$$
\mathbf{r}^{\prime}=\boldsymbol{\mu}_{\mathbf{r}^{\prime}}+\mathbf{r}
$$

with $\boldsymbol{\mu}_{\mathbf{r}^{\prime}}:=\mathrm{E}\left[\mathbf{r}^{\prime}\right], \boldsymbol{\mu}_{\mathbf{r}}:=\mathrm{E}[\mathbf{r}]=0$ and $\boldsymbol{\sigma}_{\mathbf{r}}^{2}:=\operatorname{diag}\left(\mathrm{E}\left[\mathbf{r r}^{\top}\right]\right)$.

\section{Statement of the problem}

\subsection{Generalities}

We consider a stationary non-Gaussian random aerodynamic pressure field. This field is discretized with $n_{l}$ reference points, representing for instance the pressure taps in a wind-tunnel experiment and the aerodynamic pressures are gathered in an $n_{l} \times 1$ vector $\mathbf{p}^{\prime}(t)$. We assume the mesh used for the selection of these points and the interpolation functions to reconstruct the field are selected in order to limit the admittance related to this discretization operation (Denoël and Maquoi, 2012).

The equation of motion of a structure with a linear quasi-static behavior reads

$$
\mathbf{K x}^{\prime}=\mathbf{A} \mathbf{p}^{\prime}
$$

where $\mathbf{K}$ is an $n_{u} \times n_{u}$ stiffness matrix, $\mathbf{x}^{\prime}(t)$ is an $n_{u} \times 1$ vector collecting the nodal displacements and $\mathbf{A}$ is an $n_{u} \times n_{l}$ transformation matrix mapping the aerodynamic pressures to external nodal forces.

Important quantities for the structural design, such as internal forces or stresses, referred to as structural responses are collected in an $n_{r} \times 1$ vector $\mathbf{r}^{\prime}(t)$. We only consider responses obtained by linear combinations of the aerodynamic pressures, i.e., $\mathbf{r}^{\prime}=\mathbf{B} \mathbf{p}^{\prime}$, with $\mathbf{B}$ being an $n_{r} \times n_{l}$ matrix of influence coefficients. The mean $\boldsymbol{\mu}_{\mathbf{r}^{\prime}}$ and fluctuating part $\mathbf{r}(t)$ of the response then satisfy

$$
\mu_{\mathbf{r}^{\prime}}=\mathrm{B} \mu_{\mathbf{p}^{\prime}}, \quad \mathbf{r}=\mathrm{B} \mathbf{p}, \quad \mathbf{r}^{\prime}=\boldsymbol{\mu}_{\mathbf{r}^{\prime}}+\mathbf{r} .
$$

The variance of the $i$-th structural response reads $\sigma_{\mathrm{r}_{i}}^{2}=\sum_{k=1}^{n_{l}} \sum_{l=1}^{n_{l}} \mathrm{~B}_{i k} \mathrm{~B}_{i l} \rho_{\mathrm{p}_{k} \mathrm{p}_{l}} \sigma_{\mathrm{p}_{k}} \sigma_{\mathrm{p} l}$, where $\rho_{\mathrm{p}_{k} \mathrm{p} l}$ is the correlation coefficient between the $k$-th and $l$-th components of $\mathbf{p}(t)$.

For practical design purposes, representative extreme values of $\mathbf{r}(t)$ are usually defined as the mean smallest minimum $\mathbf{r}^{(\min )}$ and the mean largest maximum $\mathbf{r}^{(\max )}$. The couple $\left(\mathbf{r}^{(\min )}, \mathbf{r}^{(\max )}\right)$ defines the envelope. The total envelope $\left(\mathbf{r}^{\prime(\min )}, \mathbf{r}^{\prime(\max )}\right)$ is then obtained by adding the mean component $\boldsymbol{\mu}_{\mathbf{r}^{\prime}}$ to the envelope $\left(\mathbf{r}^{(\min )}, \mathbf{r}^{(\max )}\right)$. 


\subsection{Determination of the envelope}

In a probabilistic framework, the envelope value associated with the $i$-th structural response is obtained by

$$
\mathrm{r}_{i}^{(\mathrm{m})}=\mathrm{g}_{i}^{(\mathrm{m})} \sigma_{\mathrm{r}_{i}}
$$

where the superscript "m" refers to either " $\min$ " or " $m a x "$ and $\mathrm{g}_{i}^{(\mathrm{m})}$ is the peak factor of the $i$-th structural response associated with either the lower $(\min )$ or upper $(\max )$ side of the envelope. The envelope value $\mathrm{r}_{i}^{(\min )}$ (resp. $\mathrm{r}_{i}^{(\max )}$ ) corresponds to the mean smallest minimum (resp. the mean largest maximum) occurring on a 10-min observation window during which the wind is considered as stationary. Under the assumption of Gaussianity, the mean smallest minimum and the mean largest maximum only differ by their sign, i.e., $\mathrm{r}_{i}^{(\min )}=-\mathrm{r}_{i}^{(\max )}$, leading to a symmetric envelope while non-Gaussian structural responses lead to an asymmetric envelope and $\mathrm{r}_{i}^{(\min )} \neq-\mathrm{r}_{i}^{(\max )}$.

For a stationary Gaussian random process and assuming that maxima occur independently of each other (Poisson assumption), Davenport (1964) gave a convenient approximation of the peak factor. In the presence of non-Gaussian excitation and nonlinear structural behavior, Winterstein (1988) formulated Hermite moment models to approximate the PDF of the random process targeting the first four cumulants. Based on this work, Kareem and Zhao (1994) derived a convenient non-Gaussian peak factor formulation that is used to compute the peak factors in this paper (Kwon and Kareem, 2011). The extension of the PSWLs concept for asymmetric envelopes undertaken in Section 4 is not tributary of the peak factor model.

\section{Conditional Expected Static Wind Loads}

\subsection{Definition}

Following common usage, the static analysis under the ESWL $\mathbf{p}^{(e, m)}$ targets one envelope value, say $\mathrm{r}_{i}^{(\mathrm{m})}$ where the superscript "e" stands for "equivalent" static wind load and " $\mathrm{m}$ " refers to either min or max envelope value. The static responses are given by

$$
\mathbf{r}^{(e, \mathrm{~m})}=\mathbf{B} \mathbf{p}^{(e, \mathrm{~m})},
$$

and, by the interpretation we give of the equivalence, the $i$-th structural response under $\mathbf{p}^{(e, m)}$ should in principle satisfy

$$
\mathrm{r}_{i}^{(e, \mathrm{~m})}=\mathrm{r}_{i}^{(\mathrm{m})}
$$

which defines the envelope value condition. In other words, an ESWL statically applied to the structure, should produce the exact envelope value of the targeted structural response.

Considering the envelope reconstruction problem, it would be desired that the static analysis under $\mathbf{p}^{(e, \mathrm{~m})}$ satisfies (5) and the following non-overestimation condition (of the envelope)

$$
\mathrm{r}_{j}^{(\min )} \leqslant \mathrm{r}_{j}^{(e, \mathrm{~m})} \leqslant \mathrm{r}_{j}^{(\max )} \quad \forall j \in\left[1, n_{r}\right] .
$$


Indeed, an ESWL derived for a specific response should not produce responses in other locations larger than their envelope values. If these two conditions are met, the reconstructed envelope obtained with a sufficiently large number of static analyses should reconstruct the real envelope, at least if all ESWLs $\left(2 n_{r}\right)$ are considered. However, depending on the formulation of ESWLs, these two conditions may not be guaranteed, as shown later. At last, the total ESWL $\mathbf{p}^{\prime(e, \mathrm{~m})}=\boldsymbol{\mu}_{\mathbf{p}^{\prime}}+\mathbf{p}^{(e, \mathrm{~m})}$ targeting one total envelope value is obtained by adding the mean wind loading $\boldsymbol{\mu}_{\mathbf{p}^{\prime}}$ to the corresponding ESWL.

The uniqueness of the equivalent static wind load is not ensured by the conditions (5)-(6) and moreover, even completely unrealistic pressure distributions may satisfy them without being plausible at all. Thinking with possible realisations of the pressure field, there exists an infinite collection of pressure distributions producing static responses satisfying the envelope value condition. To all these plausible static wind loads might be attributed a certain likelihood, which is measured here as the conditional multivariate PDF of the pressure field given the structural response $\mathrm{r}_{i}$

$$
\psi_{\mathbf{p} \mid \mathrm{r}_{i}}\left(\mathrm{p}_{1}, \ldots, \mathrm{p}_{n_{l}}, \mathrm{r}_{i}\right)=\frac{\psi_{\mathbf{p r}_{i}}\left(\mathrm{p}_{1}, \ldots, \mathrm{p}_{n_{l}}, \mathrm{r}_{i}\right)}{\psi_{\mathrm{r}_{i}}\left(\mathrm{r}_{i}\right)},
$$

where $\psi_{\mathbf{p r}_{i}}\left(\mathrm{p}_{1}, \ldots, \mathrm{p}_{n_{l}}, \mathrm{r}_{i}\right)$ is the joint $n_{l}+1$-dimensional PDF of the pressure field and the considered structural response and $\psi_{\mathrm{r}_{i}}\left(\mathrm{r}_{i}\right)$ is the marginal PDF of the considered structural response. We introduce the Conditional Expected Static Wind Load (CESWL) as the average of these plausible static wind loads conditioned upon recovery of the considered response. Mathematically, it is defined by

$$
\mathbf{p}^{(\mathcal{E}, \mathrm{m})}=\mathbb{E}\left[\mathbf{p} \mid \mathrm{r}_{i}=\mathrm{r}_{i}^{(\mathrm{m})}\right]=\mu_{\mathbf{p} \mid \mathrm{r}_{i}}\left(\mathrm{r}_{i}^{(\mathrm{m})}\right)
$$

where the symbol "E्E" stands for "conditional $\mathcal{E}$ xpected" and the $k$-th component of the conditional expected static wind load is simply obtained as

$$
\mu_{\mathrm{p}_{k} \mid \mathrm{r}_{i}}\left(\mathrm{r}_{i}^{(\mathrm{m})}\right)=\int_{\mathbb{R}} \mathrm{p}_{k} \psi_{\mathrm{p}_{k} \mid \mathrm{r}_{i}}\left(\mathrm{p}_{k}, \mathrm{r}_{i}^{(\mathrm{m})}\right) \mathrm{dp}_{k}
$$

where $\psi_{\mathrm{p}_{k} \mid \mathrm{r}_{i}}\left(\mathrm{p}_{k}, \mathrm{r}_{i}^{(\mathrm{m})}\right)$ is the conditional PDF of the $k$-th aerodynamic pressure given the $i$-th envelope value $\mathrm{r}_{i}=\mathrm{r}_{i}^{(\mathrm{m})}$. The PDF of the aerodynamic pressure $\mathrm{p}_{k}$ conditioned on the structural response $\mathrm{r}_{i}$ is obtained by a multi-fold integration of the conditional multivariate PDF (7) with respect to all other aerodynamic pressures

$$
\psi_{\mathrm{p}_{k} \mid \mathrm{r}_{i}}\left(\mathrm{p}_{k}, \mathrm{r}_{i}\right)=\int_{-\infty}^{\infty} \cdots \int_{-\infty}^{\infty} \psi_{\mathbf{p} \mid \mathrm{r}_{i}}\left(\mathrm{p}_{1}, \ldots, \mathrm{p}_{n_{l}}, \mathrm{r}_{i}\right) \mathrm{dp}_{1} \cdots \mathrm{dp}_{k-1} \mathrm{dp}_{k+1} \cdots \mathrm{dp}_{n_{l}} .
$$

As such, each component of the CESWL (9) might be derived from the sole knowledge of the conditional distribution (10) of each aerodynamic pressure given a structural response.

The loading given by (8) is such that the corresponding static response $\mathbf{r}^{(\mathcal{E}, \mathrm{m})}=\mathbf{B} \mathbf{p}^{(\mathcal{E}, \mathrm{m})}$ satisfies the envelope value condition 
$\mathrm{r}_{i}^{(\mathcal{E}, \mathrm{m})}=\sum_{k=1}^{n_{l}} \mathrm{~B}_{i k} \mathrm{p}_{k}^{(\mathcal{E}, \mathrm{m})}=\sum_{k=1}^{n_{l}} \mathrm{~B}_{i k} \mathbb{E}\left[\mathrm{p}_{k} \mid \mathrm{r}_{i}=\mathrm{r}_{i}^{(\mathrm{m})}\right]=\mathbb{E}\left[\left(\sum_{k=1}^{n_{l}} \mathrm{~B}_{i k} \mathrm{p}_{k}\right) \mid \mathrm{r}_{i}=\mathrm{r}_{i}^{(\mathrm{m})}\right]=\mathbb{E}\left[\mathrm{r}_{i} \mid \mathrm{r}_{i}=\mathrm{r}_{i}^{(\mathrm{m})}\right]=\mathrm{r}_{i}^{(\mathrm{m})}$

Also, the non-overestimation condition is an inherent feature of the conditional expected static wind load since we have

$$
\mathrm{r}_{j}^{(\min )} \leqslant \mathbb{E}\left[\mathrm{r}_{j} \mid \mathrm{r}_{i}=\mathrm{r}_{i}^{(\mathrm{m})}\right] \leqslant \mathrm{r}_{j}^{(\max )}, \quad \forall j \in\left[1, n_{r}\right]
$$

where $\mathbb{E}\left[\mathrm{r}_{j} \mid \mathrm{r}_{i}=\mathrm{r}_{i}^{(\mathrm{m})}\right]$ is the average of the $j$-th response conditioned on $\mathrm{r}_{i}=\mathrm{r}_{i}^{(\mathrm{m})}$. In a Gaussiancontext, Eq. (12) reads

$$
\mathrm{g}_{j}^{(\min )} \leqslant \mathrm{g}_{i}^{(\mathrm{m})} \rho_{\mathrm{r}_{j} \mathrm{r}_{i}} \leqslant \mathrm{~g}_{j}^{(\max )}, \quad \forall j \in\left[1, n_{r}\right] .
$$

Because of the properties (11) and (12), the envelope value and non-overestimation conditions are ipso facto fulfilled and the conditional expected static wind load introduced in (7) is a formal kind of ESWL, that is readily applicable in non-Gaussian frameworks. This observation is central to the following developments and provides the necessary information to extend the classical notions of ESWL in a non-Gaussian framework. Indeed, what Kasperski (1992) used to call "[...] the most probable extreme load [...]" could actually be understood as the average load (not the mode nor the median) conditioned upon recovery of the considered response, and, in some sense, the conditional expected static wind load extends the definition of the LRC-based ESWL into a non-Gaussian framework.

The developments aiming at the establishment of such conditional expected static wind loads (7) are classified as Conditional Expected Load (CEL) method.

\subsection{The bicubic model}

In this section, we now derive a model for the conditional load-response PDF $\psi_{\mathrm{p}_{k} \mid \mathrm{r}_{i}}\left(\mathrm{p}_{k}, \mathrm{r}_{i}\right)$ such that the non-Gaussian conditional expected SWL formulation seeks (i) consistency, the model should develop into the Gaussian formulation as a limit case, (ii) applicability, large ranges of non-Gaussianity in the random processes shall be covered, (iii) accuracy with available statistical information and (iv) simplicity of the analytical formulation. The latter ensures a computational efficiency which is required for the subsequent envelope reconstruction problem.

In this study, we consider the Hermite moment model introduced by Winterstein (1988) for the approximation of the PDF. We motivate this choice by several reasons. The model is convenient and well-known in the wind engineering community for different applications. Also, it is the cornerstone of the model for non-Gaussian peak factor developed by Kareem and Zhao (1994) which has a large applicability and accuracy. Finally, using this model for non-Gaussian peak factors, a consistent approach requires that the Hermite moment model should be kept for the PDF.

Driven by the definition we gave of the conditional expected SWL, the establishment of a nonGaussian ESWL requires the conditional PDF of the loading given an envelope value. This implies to first approximate the marginal PDFs, the joint PDF and, then, the conditional PDF of the loads and responses. 
Probability density function. We briefly review the Hermite polynomial transformation method. We model an aerodynamic pressure $\mathrm{p}$ and a structural response $\mathrm{r}$ as two cubic monotonic transformations $g(\cdot)$ and $h(\cdot)$ of two correlated standard Gaussian variables $u$ and $v$ such that

$$
\begin{aligned}
& \mathrm{p}=g(u)=\frac{\alpha_{u}}{b_{u}}\left(\frac{u^{3}}{3}+a_{u} u^{2}+\left(b_{u}-1\right) u-a_{u}\right) \\
& \mathrm{r}=h(v)=\frac{\alpha_{v}}{b_{v}}\left(\frac{v^{3}}{3}+a_{v} v^{2}+\left(b_{v}-1\right) v-a_{v}\right)
\end{aligned}
$$

where the parameters $\alpha_{u}, a_{u}, b_{u}$ are tuned to match the variance $\sigma_{\mathrm{p}}^{2}$, skewness coefficient $\gamma_{3, \mathrm{p}}$ and excess coefficient $\gamma_{e, \mathrm{p}}$ of the zero-mean random variable $\mathrm{p}$ and the parameters $\alpha_{v}, a_{v}$ and $b_{v}$ are tuned to match the variance $\sigma_{\mathrm{r}}^{2}$, skewness coefficient $\gamma_{3, \mathrm{r}}$ and excess coefficient $\gamma_{e, \mathrm{r}}$ of the zero-mean random variable r. Provided $g(u)$ and $h(v)$ are monotonic, the PDFs of the variables $\mathrm{p}$ and $\mathrm{r}$ read

$$
\psi_{\mathrm{p}}^{\mathcal{C}}(\mathrm{p})=\frac{\psi_{u}^{\mathcal{N}}(u(\mathrm{p}))}{\left|\frac{\mathrm{d} g}{\mathrm{~d} u}(u(\mathrm{p}))\right|}, \quad \psi_{\mathrm{r}}^{\mathcal{C}}(\mathrm{r})=\frac{\psi_{v}^{\mathcal{N}}(v(\mathrm{r}))}{\left|\frac{\mathrm{d} h}{\mathrm{~d} v}(v(\mathrm{r}))\right|},
$$

where the symbol "C" stands for "C Ubic model", $\psi_{u}^{\mathcal{N}}(u)=\frac{1}{\sqrt{2 \pi}} \exp \left[-u^{2} / 2\right]$ is the normal PDF (the symbol " $\mathcal{N}$ " stands for "Normal") and $u(\mathrm{p})=g^{-1}(\mathrm{p}), v(\mathrm{r})=h^{-1}(\mathrm{r}) \operatorname{read}$

$$
\begin{gathered}
u(\mathrm{p})=\left[\zeta_{u}+\sqrt{c_{u}+\zeta_{u}^{2}}\right]^{1 / 3}+\left[\zeta_{u}-\sqrt{c_{u}+\zeta_{u}^{2}}\right]^{1 / 3}-a_{u}, \\
v(\mathrm{r})=\left[\zeta_{v}+\sqrt{c_{v}+\zeta_{v}^{2}}\right]^{1 / 3}+\left[\zeta_{v}-\sqrt{c_{v}+\zeta_{v}^{2}}\right]^{1 / 3}-a_{v},
\end{gathered}
$$

with $c_{u}=\left(b_{u}-1-a_{u}^{2}\right)^{3}, \zeta_{u}(\mathrm{p})=\frac{3}{2} b_{u}\left(a_{u}+\mathrm{p} / \alpha_{u}\right)-a_{u}^{3}$ and $c_{v}=\left(b_{v}-1-a_{v}^{2}\right)^{3}, \zeta_{v}(\mathrm{r})=\frac{3}{2} b_{v}\left(a_{v}+\mathrm{r} / \alpha_{v}\right)-a_{v}^{3}$. Eq. (16) requires that the cubic transformations (14)-(15) are monotonic. This is ensured by the monotone limitations $b_{u}-1-a_{u}^{2} \geq 0$ and $b_{v}-1-a_{v}^{2} \geq 0$ (Choi and Sweetman, 2010).

The monotone limitation limits the effective region of skewness $\gamma_{3}$ and excess $\gamma_{e}$ coefficients where the approximation of random variables using the cubic transformation is applicable. In case of slight deviations from the monotone limitation, adjustments are nevertheless proposed in (Peng et al., 2014). This limitation is illustrated by the curve in Fig. 1 and the dot identifies the couple of $\left(\gamma_{3}, \gamma_{e}\right)$ for which the PDF, Eq. (16), is illustrated in Fig. 2.

Joint probability density function. The joint PDF of the aerodynamic pressure $\mathrm{p}$ and the structural response $\mathrm{r}$ modeled as two cubic monotonic transformations is given by

$$
\psi_{\mathrm{pr}}^{\mathcal{B}}(\mathrm{p}, \mathrm{r})=\frac{\psi_{u v}^{\mathcal{N}}(u(\mathrm{p}), v(\mathrm{r}))}{|J(u(\mathrm{p}), v(\mathrm{r}))|},
$$

where $\psi_{u v}^{\mathcal{N}}(u, v)=\frac{1}{2 \pi \sqrt{1-\rho_{u v}^{2}}} \exp \left[\frac{-u^{2}+2 \rho_{u v} u v-v^{2}}{2-2 \rho_{u v}^{2}}\right]$ is the Gaussian joint PDF of $u$ and $v$, the symbol "B⿱B口" stands for "Bicubic model", and $J(u, v)=\frac{\mathrm{d} g}{\mathrm{~d} u} \frac{\mathrm{d} h}{\mathrm{~d} v}$ is the Jacobian of the transformation (Papoulis, 1965). The cross-moments of the random variables $\mathrm{p}$ and $\mathrm{r}$ are defined by 


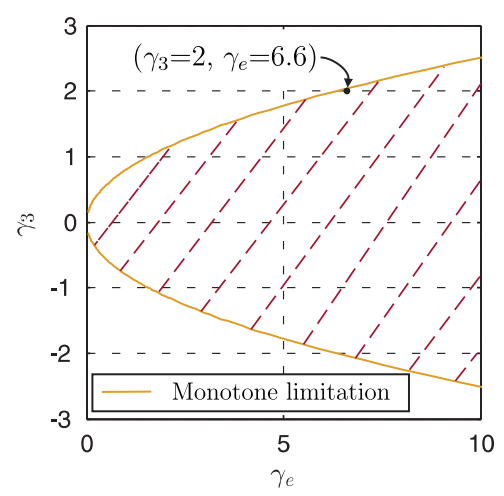

Figure 1: The domain of applicability of the Hermite moment model is the hatched area.

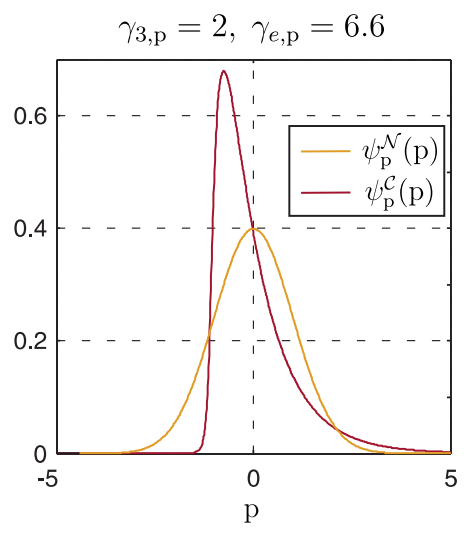

Figure 2: Gaussian PDF $\psi_{\mathrm{p}}^{\mathcal{N}}(\mathrm{p})$ and non-Gaussian PDF $\psi_{\mathrm{p}}^{\mathcal{c}}(\mathrm{p})$ obtained by the Hermite moment model, Eq. (16), of a standard random variable $\mathrm{p}$.

$$
\mathbb{E}\left[\mathrm{p}^{m} \mathrm{r}^{n}\right]=\iint_{\mathbb{R}^{2}} g(u)^{m} h(v)^{n} \psi_{u v}^{\mathcal{N}}(u, v) \mathrm{d} u \mathrm{~d} v,
$$

where $m$ and $n$ are the orders of $\mathrm{p}$ and $\mathrm{r}$, respectively. We propose to use a bicubic model with 7 parameters, $\alpha_{u}, \alpha_{v}, a_{u}, a_{v}, b_{u}, b_{v}, \rho_{u v}$. These 7 parameters are used to fit 7 statistical moments $\sigma_{\mathrm{p}}, \sigma_{\mathrm{r}}, \gamma_{3, \mathrm{p}}, \gamma_{e, \mathrm{p}}, \gamma_{3, \mathrm{r}}, \gamma_{e, \mathrm{r}}, \rho_{\mathrm{pr}}$. In our bicubic model of joint PDF (19), the correlation coefficient $\rho_{\mathrm{pr}}=$ $\mathbb{E}[\mathrm{pr}] /\left(\sigma_{\mathrm{p}} \sigma_{\mathrm{r}}\right)$ is thus the only imposed cross-moment. Actually, one could want to derive a joint PDF targeting other cross-moments. However, this is not our scope because the mathematical formulations may rapidly become complex. This explanation supports the primary choice to only target one cross-moment, through the correlation coefficient, as a compromise between accuracy and simplicity. The correlation coefficient between $\mathrm{p}$ and $\mathrm{r}$ is obtained as a function of the parameters of the bicubic model, including the correlation $\rho_{u v}$ of the two Gaussian processes $u$ and $v$, by plugging $g(u)$ and $h(v)$ into the definition of the correlation coefficient, and working out the algebra, 


$$
\begin{aligned}
\rho_{\mathrm{pr}} & =\frac{1}{\sigma_{\mathrm{p}} \sigma_{\mathrm{r}}} \iint_{\mathbb{R}^{2}} g(u) h(v) \psi_{u v}^{\mathcal{N}}(u, v) \mathrm{d} u \mathrm{~d} v \\
& =\frac{\alpha_{u} \alpha_{v}}{\sigma_{\mathrm{p}} \sigma_{\mathrm{r}}} \rho_{u v}\left(1+\frac{2 a_{u} a_{v}}{b_{u} b_{v}} \rho_{u v}+\frac{2}{3 b_{u} b_{v}} \rho_{u v}^{2}\right) .
\end{aligned}
$$

The solution of the cubic Eq. (21) in $\rho_{u v}$ reads

$$
\rho_{u v}\left(\rho_{\mathrm{pr}}\right)=\left(d+\sqrt{q+d^{2}}\right)^{1 / 3}+\left(d-\sqrt{q+d^{2}}\right)^{1 / 3}-a_{u} a_{v},
$$

where $d=\frac{3}{4} a_{u} a_{v} b_{u} b_{v}-a_{u}^{3} a_{v}^{3}+\frac{3 b_{u} b_{v} \rho_{\mathrm{pr}} \sigma_{\mathrm{p}} \sigma_{\mathrm{r}}}{4 \alpha_{u} \alpha_{v}}$ and $q=\left(\frac{b_{u} b_{v}}{2}-a_{u}^{2} a_{v}^{2}\right)^{3}$ with the condition $\frac{b_{u} b_{v}}{2}-a_{u}^{2} a_{v}^{2} \geq$ 0 .

Fig. 3 illustrates the joint PDFs computed from Eq. (19) for correlation coefficients $\rho_{\text {pr }}$ equal to 0 and 0.5 , and for three sets of $\left(\gamma_{3}, \gamma_{e}\right)$. Figs. 3(a) and (d) shows limit cases where the variables are Gaussian. The intermediate case of joint PDFs of one normal variable and another non-Gaussian is illustrated in Figs. 3(b) and (e). For two non-Gaussian variables, the joint PDFs are drawn in Figs. 3(c) and (f). These illustrations show that a wide variety of joint PDF might be spanned by this bicubic transformation method.
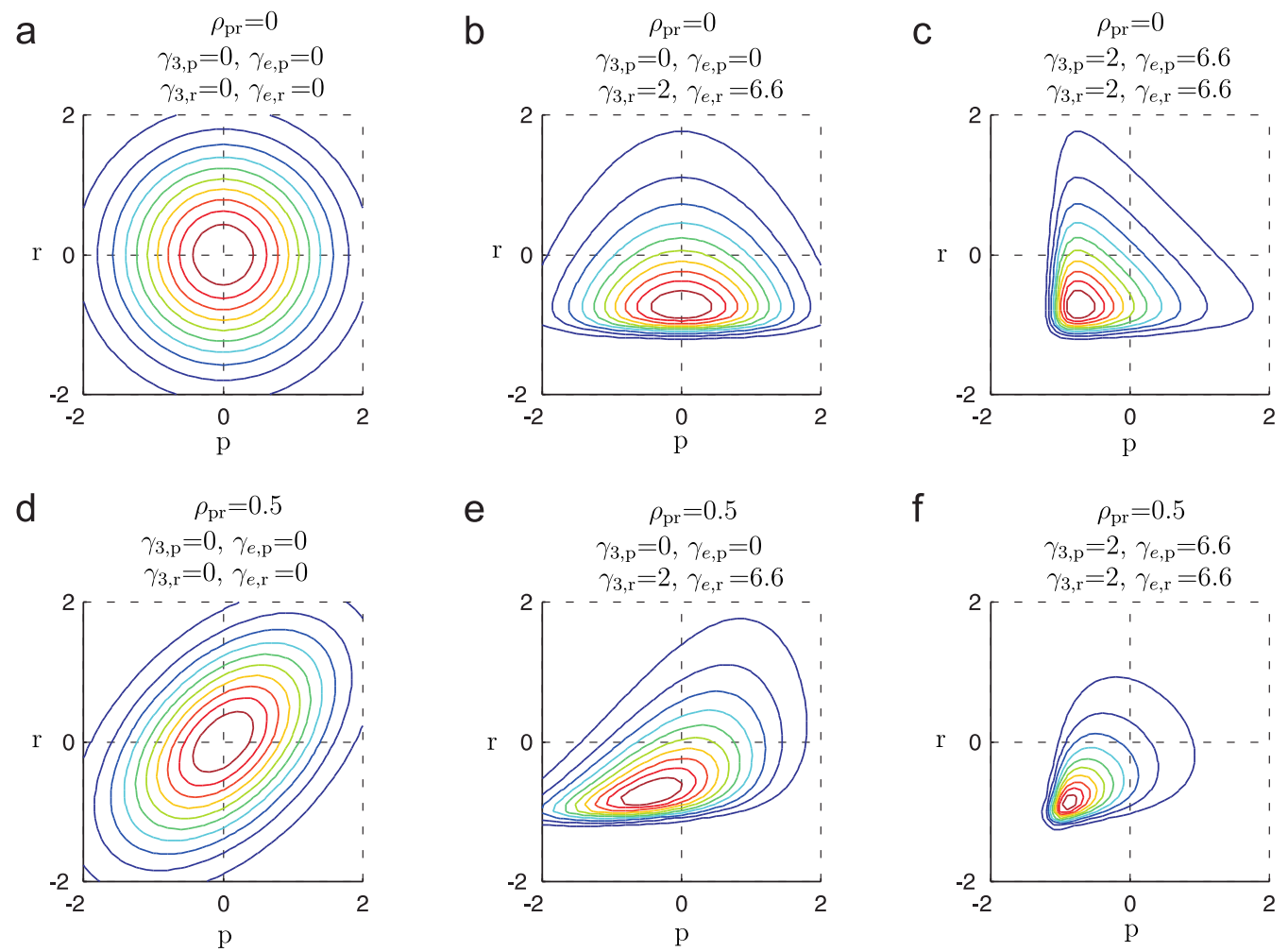

Figure 3: Joint PDFs $\psi_{\mathrm{pr}}^{\mathcal{B}}(\mathrm{p}, \mathrm{r})$ obtained by the bicubic model, Eq. (19), of two standard random variables $\mathrm{p}$ and $\mathrm{r}$ for different couples $\left(\gamma_{3}, \gamma_{e}\right)$ and two correlation coefficients 0 and 0.5 . 
Conditional probability density function. The conditional PDF of $\mathrm{p}$ given $\mathrm{r}$ is written

$$
\psi_{\mathrm{p} \mid \mathrm{r}}^{\mathcal{B}}(\mathrm{p}, \mathrm{r})=\frac{\psi_{u \mid v}^{\mathcal{N}}(u(\mathrm{p}), v(\mathrm{r}))}{\left|\frac{\mathrm{d} g}{\mathrm{~d} u}(u(\mathrm{p}))\right|},
$$

where $\psi_{u \mid v}^{\mathcal{N}}(u, v)=\frac{1}{\sqrt{1-\rho_{u v}^{2}} \sqrt{2 \pi}} \exp \left[-\left(u-v \rho_{u v}\right)^{2} /\left(2-2 \rho_{u v}^{2}\right)\right]$ is the Gaussian joint PDF of $u$ given $v$. The conditional mean of $\mathrm{p}$ given $\mathrm{r}$ is obtained by

$$
\begin{aligned}
\mu_{\mathrm{p} \mid \mathrm{r}}^{\mathcal{B}}(\mathrm{r}) & =\int_{\mathbb{R}} g(u) \psi_{u \mid v}^{\mathcal{N}}(u(\mathrm{p}), v(\mathrm{r})) \mathrm{d} u \\
& =\frac{\alpha_{u}}{3 b_{u}}\left(\left(v(\mathrm{r})^{3}-3 v(\mathrm{r})\right) \rho_{u v}^{3}+3 a_{u}\left(v(\mathrm{r})^{2}-1\right) \rho_{u v}^{2}+3 b_{u} v(\mathrm{r}) \rho_{u v}\right) .
\end{aligned}
$$

For standard variables $\mathrm{p}$ and r, Fig. 4 illustrates the conditional PDFs of $\mathrm{p}$ given $\mathrm{r}=3.5$ computed from Eq. (23) with a correlation coefficient $\rho_{\mathrm{pr}}=0.5$. Two intermediate cases are shown in Fig. 4: (a) the random variable $\mathrm{p}$ is normal and $\mathrm{r}$ is not and (b) the random variable $\mathrm{p}$ is non-Gaussian and $\mathrm{r}$ is normal. Fig. 4(c) illustrates the case for two non-Gaussian random variables. Significant differences between the conditional mean value obtained through the bicubic model $\mu_{\mathrm{p} \mid \mathrm{r}}^{\mathcal{B}}(3.5)$ and the Gaussian one, $\mu_{\mathrm{p} \mid \mathrm{r}}^{\mathcal{N}}(3.5)=\mathrm{r} \rho_{\mathrm{pr}}=1.75$, can be observed in Fig. 4 .

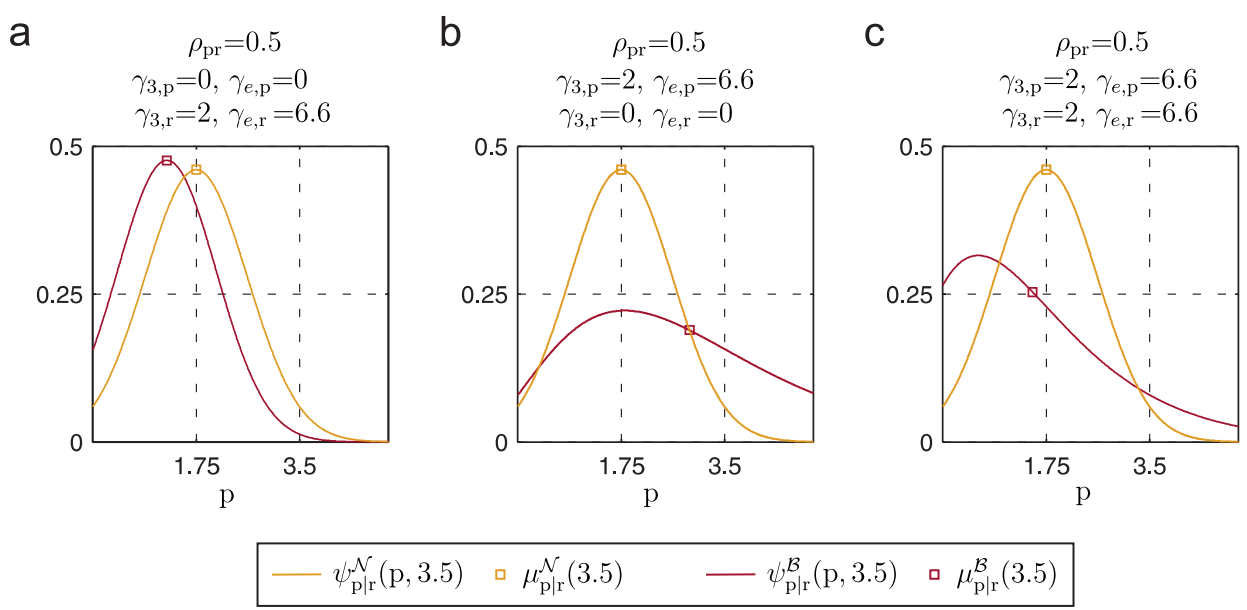

Figure 4: Gaussian conditional PDF $\psi_{\mathrm{p} \mid \mathrm{r}}^{\mathcal{N}}(\mathrm{p}, \mathrm{r})$ and non-Gaussian conditional PDF $\psi_{\mathrm{p} \mid \mathrm{r}}^{\mathcal{B}}(\mathrm{p}, \mathrm{r})$ obtained by the bicubic model, Eq. (23), of p given $\mathrm{r}=3.5$ for different couples $\left(\gamma_{3}, \gamma_{e}\right)$ and a correlation coefficient $\rho_{\mathrm{pr}}=0.5$.

Formulation of the conditional expected SWL with the bicubic model. The $k$-th loading component of the $\operatorname{ESWL}\left(\mathbf{p}^{(e, \mathbf{m})}=\mathbf{p}^{(\mathcal{B}, \mathbf{m})}\right)$ that targets the $i$-th envelope value $\mathrm{r}_{i}^{(\mathrm{m})}$ is defined as

$$
\mathrm{p}_{k}^{(\mathcal{B}, \mathrm{m})}=\mu_{\mathrm{p}_{k} \mid \mathrm{r}_{i}}^{\mathcal{B}}\left(\mathrm{r}_{i}^{(\mathrm{m})}\right)
$$

where $\mu_{\mathrm{p}_{k} \mid \mathrm{r}_{i}}^{\mathcal{B}}\left(\mathrm{r}_{i}^{(\mathrm{m})}\right)$ is given by Eq. (24) with $\mathrm{p} \equiv \mathrm{p}_{k}$ and $\mathrm{r} \equiv \mathrm{r}_{i}$ given $\mathrm{r}_{i}=\mathrm{r}_{i}^{(\mathrm{m})}$. The ESWL given by (25) is an approximation of the exact conditional expected SWL (7), i.e., $\mathrm{p}_{k}^{(\mathcal{B}, \mathrm{m})} \approx \mathrm{p}_{k}^{(\mathcal{E}, \mathrm{m})}$, since the joint 
and conditional PDFs are approximations through the proposed bicubic model of the formal ones. As a consequence, the envelope value (5) and non-overestimation (12) conditions may be not fulfilled, as studied in the illustrations.

In case both variables $\mathrm{p}$ and $\mathrm{r}$ are Gaussian random variables, the parameters of the cubic transformations become

$$
\alpha_{u} \rightarrow \sigma_{\mathrm{p}}, \alpha_{v} \rightarrow \sigma_{\mathrm{r}}, b_{u} \rightarrow \infty, b_{v} \rightarrow \infty, a_{u} \rightarrow 0, a_{v} \rightarrow 0
$$

which yields $\rho_{\text {pr }}=\rho_{u v}$ in Eq. (22) and in Eq. (24) the conditional mean value of p given $\mathrm{r}$ becomes

$$
\mu_{\mathrm{p} \mid \mathrm{r}}^{\mathcal{N}}(\mathrm{r})=\frac{\mathrm{r}}{\sigma_{\mathrm{r}}} \rho_{\mathrm{pr}} \sigma_{\mathrm{p}}
$$

Finally the $k$-th loading component expressed by Eq (25) degenerates into the LRC formulation

$$
\mathrm{p}_{k}^{(\mathcal{L}, \mathrm{m})}=\mu_{\mathrm{p}_{k} \mid \mathrm{r}_{i}}^{\mathcal{N}}\left(\mathrm{r}_{i}^{(\mathrm{m})}\right)=\frac{\mathrm{r}_{i}^{(\mathrm{m})}}{\sigma_{\mathrm{r}_{i}}} \rho_{\mathrm{p}_{k} \mathrm{r}_{i}} \sigma_{\mathrm{p}_{k}}
$$

as a limit case, where the symbol " $\mathcal{L}$ " stands for "Load"-response correlation method. In a Gaussian context, the ESWL given by $(28)$ is the exact conditional expected SWL (7), i.e., $\mathrm{p}_{k}^{(\mathcal{L}, \mathrm{m})}=\mathrm{p}_{k}^{(\mathcal{E}, \mathrm{m})}$, since the joint and conditional PDFs are Gaussian ones and no approximation is made. The envelope value (5) and non-overestimation (13) conditions are therefore fulfilled with the LRC-based ESWLs in a Gaussian context. If the LRC-based ESWLs are used in case of non-Gaussianities along with "non-Gaussian" peak factors, the envelope value condition is still satisfied since

$$
\mathrm{r}_{i}^{(\mathrm{m})}=\mathrm{g}_{i}^{(\mathrm{m})} \sigma_{\mathrm{r}_{i}}=\sum_{k=1}^{n_{l}} \mathrm{~B}_{i k}\left(\mathrm{~g}_{i}^{(\mathrm{m})} \sum_{l=1}^{n_{l}} \mathrm{~B}_{i l} \rho_{\mathrm{p}_{k} \mathrm{p}_{l}} \sigma_{\mathrm{p}_{k}} \sigma_{\mathrm{p}_{l}} / \sigma_{\mathrm{r}_{i}}\right),
$$

and the term in parenthesis corresponds to the LRC-based ESWLs with $\rho_{\mathrm{p}_{k} \mathrm{r}_{i}}=\sum_{l=1}^{n_{l}} \mathrm{~B}_{i l} \rho_{\mathrm{p}_{k} \mathrm{p}_{l}} \sigma_{\mathrm{p}_{l}} / \sigma_{\mathrm{r}_{i}}$. This demonstrates that the envelope value condition is not a sufficient condition to ensure that the ESWL is the conditional expected one.

\subsection{Conditional sampling technique}

Common practice consists in identifying extreme values of structural responses on each observation window and sampling the associated pressure distributions (Holmes, 1988; Tamura et al., 2002). The common CST-based ESWL $\left(\mathbf{p}^{(e, m)}=\mathbf{p}^{(\mathcal{S}, \mathrm{m})}\right)$ is then defined as the statistical average of these sampled load patterns

$$
\mathbf{p}^{(\mathcal{S}, \mathrm{m})}=\operatorname{mean}_{n_{T}} \hat{\mathbf{p}}\left(t_{k}\right), k \in\left[1, \ldots, n_{T}\right]
$$

where $\hat{\mathbf{p}}\left(t_{k}\right)$ is the $k$-th load pattern associated with the extreme value $\hat{r}_{i}$ of a structural response on the $k$-th observation window and occurring at time $t_{k}$. The average is made on load patterns producing the extreme values on each observation window, each one being slightly different from the envelope value, i.e., $\hat{\mathrm{r}}_{i} \neq \mathrm{r}_{i}^{(\mathrm{m})}$.

In a statistical framework, where time series are available, envelope values of structural responses may also be obtained with inferential statistics on realizations. The observed extreme values of the 
$i$-th structural response are identified on each observation window and their average, tends to the exact envelope value, denoted $\mathbb{E}\left[\hat{\mathrm{r}}_{i}\right]$, as the number of observation windows increases.

In a Gaussian framework, the CESWL $\mathbf{p}^{(\mathcal{E}, \mathrm{m})}$ associated with the $i$-th structural response is linear with respect to the value given to $\mathrm{r}_{i}$, see Eq. (27). Hence, if the envelope value computed is the exact one, i.e., $\mathrm{r}_{i}^{(\mathrm{m})}=\mathbb{E}\left[\hat{\mathrm{r}}_{i}\right]$, the formulation given by Eq. (30) tends to the conditional expected SWL as the number of observation window increases, i.e., $\mathbf{p}^{(\mathcal{S}, \mathrm{m})} \rightarrow \mathbf{p}^{(\mathcal{E}, \mathrm{m})}$.

In a non-Gaussian framework, the CESWL $\mathbf{p}^{(\mathcal{E}, \mathrm{m})}$ associated with the $i$-th structural response is nonlinear with respect to the value given to $r_{i}$ and thus the loading $\mathbf{p}^{(\mathcal{S}, m)}$ does not converge toward the conditional expected SWL as the number of observation window increases, i.e., $\mathbf{p}^{(\mathcal{S}, \mathrm{m})} \nrightarrow \mathbf{p}^{(\mathcal{E}, \mathrm{m})}$, even if the peak factor is the exact one. The more the number of observation windows are considered, the more the envelope value condition (5) is fulfilled while the non-overestimation (12) condition is not naturally fulfilled, as studied in the illustrations.

\subsection{Two-step adjustment method}

The above developments have shown that the equivalent static wind loads $\mathbf{p}^{(e, \mathrm{~m})}$ with $e \equiv \mathcal{L}$ (LRC), $e \equiv \mathcal{S}$ (common conditional sampling) or $e \equiv \mathcal{B}$ (bicubic model), do not necessarily satisfy the envelope value condition, nor the non-overestimation condition.

If the envelope value condition is not fulfilled under the original ESWLs $\mathbf{p}^{(e, \mathrm{~m})}$, scaled ESWLs $\tilde{\mathbf{p}}^{(e, \mathrm{~m})}$ are defined for this purpose by

$$
\tilde{\mathbf{p}}^{(e, \mathrm{~m})}=\alpha^{(e, \mathrm{~m})} \mathbf{p}^{(e, \mathrm{~m})},
$$

where the scaling coefficient $\alpha^{(e, \mathbf{m})}$ is determined to ensure the envelope value condition. Should the fulfillment of the non-overestimation condition fail under scaled ESWLs, it is always possible to define adjusted ESWLs $\breve{\mathbf{p}}^{(e, \mathrm{~m})}$, satisfying the 2 conditions, by

$$
\breve{\mathbf{p}}^{(e, \mathrm{~m})}=\boldsymbol{\beta}^{(e, \mathrm{~m})} \circ \alpha^{(e, \mathrm{~m})} \mathbf{p}^{(e, \mathrm{~m})},
$$

where the local coefficients $\boldsymbol{\beta}^{(e, \mathrm{~m})}$, an $n_{l} \times 1$ vector, adjust the scaled ESWLs $\tilde{\mathbf{p}}^{(e, \mathrm{~m})}$ in order to fulfill the non-overestimation condition. These local coefficients should slightly increase or decrease the components of the ESWL without distorting too much the scaled ESWL. These coefficients have to be as close to unity as possible but make sure the non-overestimation condition is met. Computation of the local coefficients vector $\boldsymbol{\beta}^{(e, \mathrm{~m})}$ is formalized here as a constrained nonlinear optimization. We want to hold the envelope value condition and to satisfy the non-overestimation condition by finding the minimum of a problem specified by

$$
\min _{\boldsymbol{\beta}^{(e, \mathrm{~m})}} \sum_{j=1}^{n_{l}}\left|\beta_{j}^{(e, \mathrm{~m})}-1\right|^{\gamma}
$$

under the linear constraints

$$
\begin{cases}+\mathbf{B} \breve{\mathbf{p}}^{(e, \mathrm{~m})}-\mathbf{r}^{(\max )} & \leqslant \mathbf{0}, \\ -\mathbf{B} \breve{\mathbf{p}}^{(e, \mathrm{~m})}+\mathbf{r}^{(\mathrm{min})} & \leqslant \mathbf{0}, \\ \sum_{j=1}^{n_{l}} \mathrm{~B}_{i j} \breve{\mathrm{p}}_{j}^{(e, \mathrm{~m})} & =\mathrm{r}_{i}^{(\mathrm{m})}\end{cases}
$$


The first two constraints in Eq. (34) are the non-overestimation condition while the third one corresponds to the envelope value condition. Symbol $\gamma$ is a positive coefficient taken here equal to 2 as a compromise between accuracy and convergence of the optimization. The magnitudes of $\alpha^{(e, m)}$ and $\boldsymbol{\beta}^{(e, m)}$ are used in the illustrations to compare ESWL formulations and identify optimal ones.

\section{Principal static wind loads}

\subsection{Extension in a non-Gaussian framework}

This section provides a generalized definition of (the basis) of PSWLs for non-Gaussian structural responses. It must be emphasized that this new definition is in accordance with the one given in (Blaise and Denoël, 2013) in the particular case of Gaussian structural responses.

The $n_{l} \times n_{r}$ ESWL matrices $\mathbf{P}^{(e, \min )}$ and $\mathbf{P}^{(e, \max )}$ consist of columns collecting the ESWLs $\mathbf{p}^{(e, \mathbf{m})}$ , with $e \in\{\mathcal{L}, \mathcal{S}, \mathcal{B}\}$, computed for all envelope values, minima and maxima, respectively. We define an $n_{l} \times 2 n_{r}$ matrix $\mathbf{P}^{(e)}$ collecting all ESWLs

$$
\mathbf{P}^{(e)}=\left[\mathbf{P}^{(e, \min )} \mathbf{P}^{(e, \max )}\right]
$$

The PSWLs are defined as the columns of the principal matrix $\mathbf{P}^{(p)}$ resulting from the singular value decomposition (SVD) of $\mathbf{P}^{(e)}$

$$
\mathbf{P}^{(e)}=\mathbf{P}^{(p)} \mathbf{S V}^{\prime}
$$

The main diagonal of $\mathbf{S}$ gathers the principal coordinates, while $\mathbf{V}$ collects the combination coefficients to reconstruct the matrix $\mathbf{P}^{(e)}$. PSWLs are ordered by decreasing variance and only the first few modes are retained for the envelope reconstruction problem (Blaise and Denoël, 2013).

The PSWLs, derived from Eq. (36), require normalization before the envelope reconstruction. Two normalized PSWLs, $\mathbf{P}_{i}^{(p, 1)}$ and $\mathbf{P}_{i}^{(p, 2)}$, are defined for each PSWL by

$$
\mathbf{P}_{i}^{(p, 1)}=\alpha_{i}^{(p, 1)} \mathbf{P}_{i}^{(p)}, \quad \quad \mathbf{P}_{i}^{(p, 2)}=\alpha_{i}^{(p, 2)}\left(-\mathbf{P}_{i}^{(p)}\right)
$$

where $\alpha_{i}^{(p, 1)}$ and $\alpha_{i}^{(p, 2)}$ are two positive coefficients applied to satisfy the tangency condition. Note that in a Gaussian framework: $\alpha_{i}^{(p, 1)}=\alpha_{i}^{(p, 2)}$. The tangency condition is such that the $i$-th static response $\mathbf{R}_{i}^{(p, 1)}=\mathbf{B P}_{i}^{(p, 1)}$ under a PSWL is somewhere tangent to the envelope, while there is no overestimation anywhere else. The same definition holds for $\mathbf{R}_{i}^{(p, 2)}$. Mathematically, we may write

$$
\begin{array}{ll}
\forall i, & \left(\exists j \in\left[1, n_{r}\right]: \quad \mathrm{R}_{j i}^{(p, 1)}=\mathrm{r}_{j}^{(\max )} \quad \text { or } \mathrm{R}_{j i}^{(p, 1)}=\mathrm{r}_{j}^{(\min )}\right) \\
\wedge & \left(\mathrm{r}_{j}^{(\min )} \leqslant \mathrm{R}_{j i}^{(p, 1)} \leqslant \mathrm{r}_{j}^{(\max )} \forall j \in\left[1, n_{r}\right]\right) .
\end{array}
$$

PSWLs are not derived from adjusted ESWLs but from the original ones. Indeed, even if ESWLs do not fulfill the envelope value and non-overestimation conditions, PSWLs anyway have to be normalized to fulfill the tangency condition. Furthermore in case of large non-Gaussianity, adjusted loadings may be significantly different from the original ones; this is shown later in the illustrations. However, both ESWL bases have been investigated by the authors and it was observed that the efficiency of the PSWLs for the envelope reconstruction problem is sometimes better, sometimes worse, considering 
adjusted ESWLs rather than the original ones for the SVD operation. Besides, this positive or negative impact on the envelope reconstruction is rather low. The demanding computation of the local coefficients $\boldsymbol{\beta}^{(e, \mathrm{~m})}$ is therefore not justified. Thence we recommend the use of the original ESWLs for computing PSWLs and only this approach is illustrated in the present paper.

\subsection{Envelope reconstruction without combinations}

The sequential reconstruction $\left(\tilde{\mathbf{r}}_{(k)}^{(\min )}, \tilde{\mathbf{r}}_{(k)}^{(\max )}\right)$ of the envelope $\left(\mathbf{r}^{(\min )}, \mathbf{r}^{(\max )}\right)$ after considering the first $k$ normalized PSWLs is expressed by the recursive relations for $k$ odd

$$
\begin{aligned}
& \tilde{\mathbf{r}}_{(k)}^{(\max )}=\max \left(\tilde{\mathbf{r}}_{(k-1)}^{(\max )} ; \mathbf{R}_{\frac{k+1}{2}}^{(p, 1)}\right), \\
& \tilde{\mathbf{r}}_{(k)}^{(\min )}=\min \left(\tilde{\mathbf{r}}_{(k-1)}^{(\min )} ; \mathbf{R}_{\frac{k+1}{2}}^{(p, 1)}\right) \text {, } \\
& \tilde{\mathbf{r}}_{(k+1)}^{(\max )}=\max \left(\tilde{\mathbf{r}}_{(k)}^{(\max )} ; \mathbf{R}_{\frac{k+1}{2}}^{(p, 2)}\right), \\
& \tilde{\mathbf{r}}_{(k+1)}^{(\min )}=\min \left(\tilde{\mathbf{r}}_{(k)}^{(\min )} ; \mathbf{R}_{\frac{k+1}{2}}^{(p, 2)}\right) \text {, }
\end{aligned}
$$

with $\tilde{\mathbf{r}}_{(0)}^{(\mathrm{m})}=\mathbf{0}$. Furthermore, the total envelope $\left(\mathbf{r}^{\prime(\min )}, \mathbf{r}^{\prime(\max )}\right)$ is approximated by adding to $\left(\tilde{\mathbf{r}}_{(k)}^{(\min )}, \tilde{\mathbf{r}}_{(k)}^{(\max )}\right)$ the vector of mean responses $\boldsymbol{\mu}_{\mathbf{r}^{\prime}}$.

\subsection{Envelope reconstruction with combinations}

Combining a reduced set of PSWLs performs better for the envelope reconstruction than applying successively each normalized PSWL separately (Blaise et al., 2012). For these reasons, only the first few $n_{p}$ PSWLs are retained and combinations of them are considered instead

$$
\mathbf{p}_{(k)}^{(c)}=\left[\mathbf{P}_{1}^{(p)}, \mathbf{P}_{2}^{(p)}, \ldots, \mathbf{P}_{n_{p}}^{(p)}\right] \mathbf{q}_{(k)}^{(c)},
$$

with $\mathbf{q}_{(k)}^{(c)}$ an $n_{p} \times 1$ vector of combination coefficients and $\mathbf{p}_{(k)}^{(c)}$ an $n_{l} \times 1$ vector representing a static wind load obtained by combinations of the first $n_{p}$ PSWLs. The combination coefficients in $\mathbf{q}_{(k)}^{(c)}$ are such that the static responses $\mathbf{r}_{(k)}^{(c)}=\mathbf{B} \mathbf{p}_{(k)}^{(c)}$, associated with the combinations of PSWLs satisfy the tangency condition. The envelope reconstruction problem requires the determination of a sufficient number of PSWLs and combinations thereof, such that the envelope $\tilde{\mathbf{r}}_{(k)}^{(\mathrm{m})}$ of the structural responses be close enough — within an a priori given accuracy, and metrics - to the actual envelope $\mathbf{r}^{(\mathrm{m})}$. This reconstruction of the envelope may be seen in an iterative manner, seeking at each step the combination that will appropriately fill the gaps between the actual envelope and the envelope reconstructed with the former iterations.

At the $k$-th iteration, the sequential reconstruction $\left(\tilde{\mathbf{r}}_{(k)}^{(\min )}, \tilde{\mathbf{r}}_{(k)}^{(\max )}\right)$ of the envelope $\left(\mathbf{r}^{(\min )}, \mathbf{r}^{(\max )}\right)$ is expressed by the recursive relations

$$
\begin{aligned}
& \tilde{\mathbf{r}}_{(k)}^{(\min )}=\min \left(\tilde{\mathbf{r}}_{(k-1)}^{(\min )} ; \mathbf{r}_{(k)}^{(c)}\right) ; \\
& \tilde{\mathbf{r}}_{(k)}^{(\max )}=\max \left(\tilde{\mathbf{r}}_{(k-1)}^{(\max )} ; \mathbf{r}_{(k)}^{(c)}\right) \text {. }
\end{aligned}
$$


The relative errors between the envelope and its reconstruction are estimated by

$$
\varepsilon_{(k)}^{(m)}=\left(\tilde{\mathbf{r}}_{(k)}^{(\mathrm{m})}-\mathbf{r}^{(\mathrm{m})}\right) \div \mathbf{r}^{(\mathrm{m})},
$$

where division is performed element by element.

The combination coefficients $\mathbf{q}_{(k)}^{(c)}$ are determined with a constrained nonlinear optimization problem chosen as

$$
\min _{\substack{(c) \\ \mathbf{q}_{(k)}}} f\left(\varepsilon_{(k)}^{(\mathrm{m})}\right)=\left\|\varepsilon_{(k)}^{(\min )}\right\|_{1}+\left\|\varepsilon_{(k)}^{(\max )}\right\|_{1}
$$

under the linear constraints of the non-overestimation conditions

$$
\left\{\begin{array}{cc}
+\mathbf{B}\left[\mathbf{P}_{1}^{(p)}, \mathbf{P}_{2}^{(p)}, \ldots, \mathbf{P}_{n_{p}}^{(p)}\right] \mathbf{q}_{(k)}^{(c)} & -\mathbf{r}^{(\max )} \leqslant \mathbf{0} \\
-\mathbf{B}\left[\mathbf{P}_{1}^{(p)}, \mathbf{P}_{2}^{(p)}, \ldots, \mathbf{P}_{n_{p}}^{(p)}\right] \mathbf{q}_{(k)}^{(c)} & +\mathbf{r}^{(\min )} \leqslant \mathbf{0} .
\end{array}\right.
$$

The cost function (43) to be minimized aims at the minimization of the relative reconstruction errors.

A procedure to guess a good initial set of combination coefficients to start the optimization is described. First, each retained PSWL for combinations is multiplied by coefficients chosen in the triplet $\left\{-\alpha_{i}^{(p, 2)}, 0, \alpha_{i}^{(p, 1)}\right\}$. Second, all possible combinations of each coefficient of PSWL are considered. Discarding the trivial combination, for $n_{p}$ PSWLs, the number of possible combinations amounts to $3^{n_{p}}-1$. For instance, for $n_{p}=2$ PSWLs, the 8 sets of combination coefficients are given by each column of the matrix below

$$
\left[\begin{array}{cccccccc}
-\alpha_{1}^{(p, 2)} & -\alpha_{1}^{(p, 2)} & -\alpha_{1}^{(p, 2)} & 0 & 0 & \alpha_{1}^{(p, 1)} & \alpha_{1}^{(p, 1)} & \alpha_{1}^{(p, 1)} \\
-\alpha_{2}^{(p, 2)} & 0 & \alpha_{2}^{(p, 1)} & -\alpha_{2}^{(p, 2)} & \alpha_{2}^{(p, 1)} & -\alpha_{2}^{(p, 2)} & 0 & \alpha_{2}^{(p, 1)}
\end{array}\right] .
$$

Third, each set of combination coefficients (each column above) is scaled by a scalar to fulfill the tangency condition. Fourth, the cost function is evaluated for each set of combination coefficients and the set providing the minimum cost function is selected as the initial value $\mathbf{q} \mathbf{0}_{(k)}$ for the optimization. After optimization, the reconstructed envelope is updated and we proceed to the next iteration. We recommend the sequential quadratic programming method for this constrained nonlinear optimization.

\section{Illustrations}

A rigid gable-roofed low-rise building is analyzed under wind actions. Extensive wind-tunnel tests have been performed to study the aerodynamic pressure field on these usual structures. For normal dimensions, they are common to build for many applications, mainly residential and industrial. The structure used for illustrations has sharp edges between the vertical walls and the roof. Depending on the angle of attack of the wind, the aerodynamic pressure field for this roof configuration is known to exhibit mildly to strongly non-Gaussianities. The roof is supported by a collection of frames and we focus on two specific ones for the illustrations. 


\subsection{Wind tunnel tests}

The dimensions of the structure are a width of $36.6 \mathrm{~m}(120 \mathrm{ft})$, an eave height of $3.65 \mathrm{~m}(12 \mathrm{ft})$, a length of $57.2 \mathrm{~m}(187.5 \mathrm{ft})$ and a roof slope of 1:12. Wind-tunnel measurements have been done at the Boundary Layer Wind Tunnel Laboratory of the University of Western Ontario (Ho et al., 2005), see the scaled model in Fig. 5.

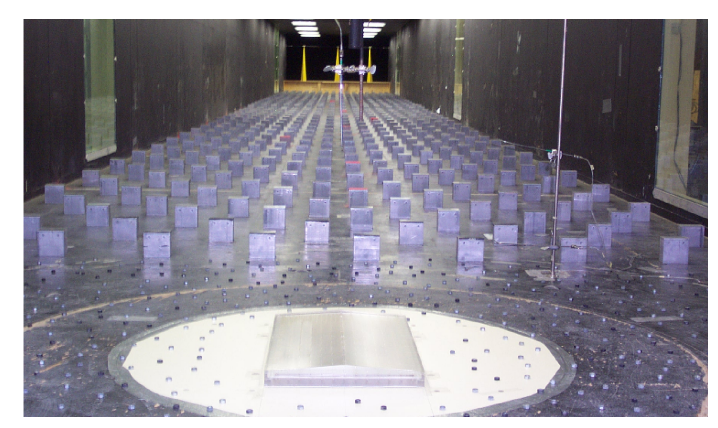

Figure 5: Picture of the rigid gable-roofed building in the wind-tunnel. From windPressure (Main and Fritz, 2006).

The pressure time series are available from (Main, 2006) and can be used through windPressure (Main and Fritz, 2006), a Matlab-based Database-Assisted Design software available on the internet. The length scale is 1:100, the sampling frequency in the wind-tunnel is $500 \mathrm{~Hz}$ and the terrain condition is suburban, corresponding to a roughness length of $0.3 \mathrm{~m}$. The mean wind speed at eave height is equal to $5.91 \mathrm{~m} / \mathrm{s}$ in the wind tunnel. In full scale, the mean wind speed $V$ at eave height is equal to $14.73 \mathrm{~m} / \mathrm{s}$ and the velocity and time scales are $1 / 2.5$ and $1 / 40.1$, respectively. The sampling frequency corresponds to $12.5 \mathrm{~Hz}$ in full scale (a time step equal to 0.08 seconds). Each measurement lasts about 66.6 minutes full scale. The leakage case is no opening in the building.

Pressure coefficients are usually referenced using the mean wind speed at a reference height $V$; they are defined from the aerodynamic pressures by

$$
\mathbf{c}_{\mathbf{p}}^{\prime}=\frac{1}{1 / 2 \rho V^{2}} \mathbf{p}^{\prime}
$$

along with

$$
\mathbf{c}_{\mathbf{p}}^{(e, \mathrm{~m})}=\frac{1}{1 / 2 \rho V^{2}} \mathbf{p}^{(e, \mathrm{~m})}, \quad \mathbf{C}_{\mathbf{p}}^{(p)}=\frac{1}{1 / 2 \rho V^{2}} \mathbf{P}^{(p)}
$$

and where $\rho=1.225 \mathrm{~kg} / \mathrm{m}^{3}$ is the air density. A positive pressure coefficient means aerodynamic pressure acts towards the inner of the building while a negative coefficient indicates suction (with reference to the atmospheric pressure). For convenience, ESWLs as well as PSWLs are illustrated with the pressure coefficients.

Fig. 6 shows, in an exploded view, the tap array (indicated by the dots) as well as tributary areas for each pressure tap $\left(n_{l}=395\right)$ on the vertical faces along the length of the building and on the roof.

The vertical faces and the two slopes of the roof are slightly shifted each other for clarity. Taps on the vertical faces along the width of the building are disregarded in the structural analysis and are thus not shown in Fig. 6. The wind direction $\theta=340^{\circ}$ is chosen for illustration, see Fig. 6. 


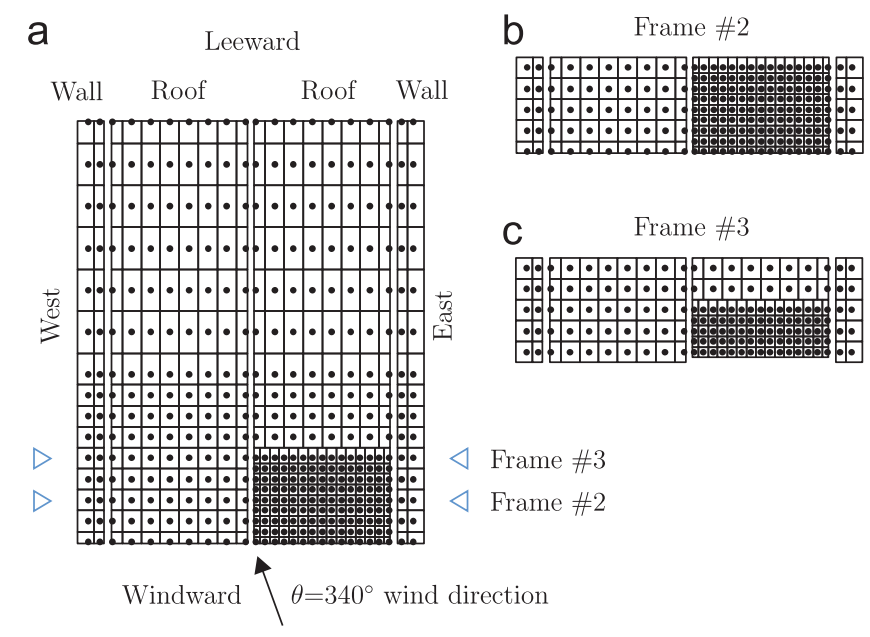

Figure 6: (a) Exploded view of the tap array with varying tap density, the triangles identify two frames considered for illustrations. Only aerodynamic pressures measured at the taps contributing to the reaction forces of the girts and purlins attached to (b) Frame \#2 and (c) Frame \#3 are used, respectively, for illustrations. The wind direction $\theta=340^{\circ}$ is studied.

Fig. 7 shows the maps of the mean and standard deviations along with the skewness and excess coefficients of the pressure coefficients.

The roof is mainly loaded close to the sharp edge roof connection with the gable end about a quarter of the length of the building, along Frame \#2. The mean loading is suction and large standard deviations close to the gable end is explained by the flow detachment intensity which is important on this windward side of the roof since the air flow encounters the structure's roof with its sharp edge connection between the horizontal and vertical parts. The aerodynamic pressure field exhibits also large skewness and excess coefficients, up to -2 and 8, respectively. This must be taken into account in a non-Gaussian analysis.

a

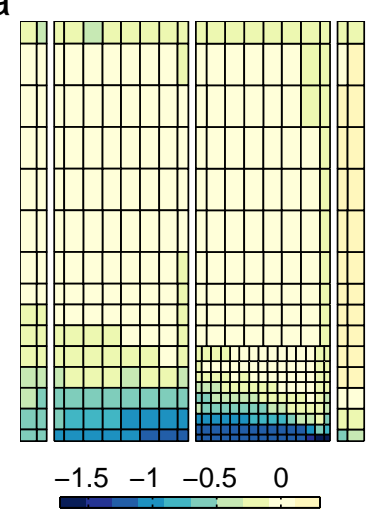

b

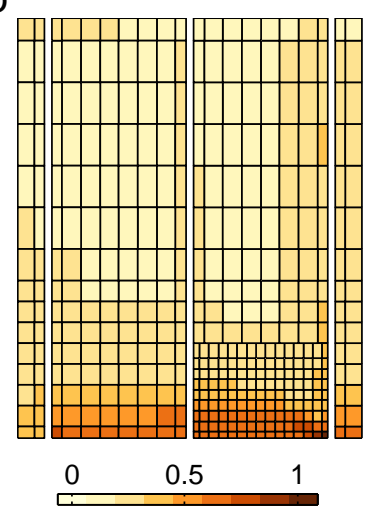

C

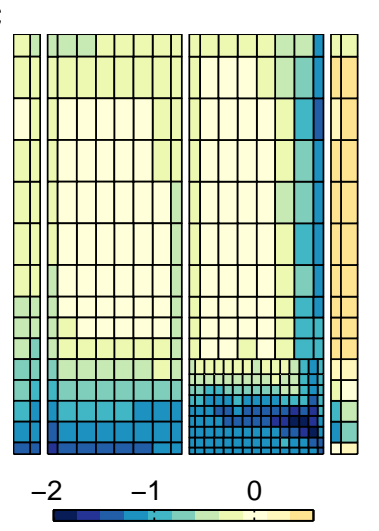

d

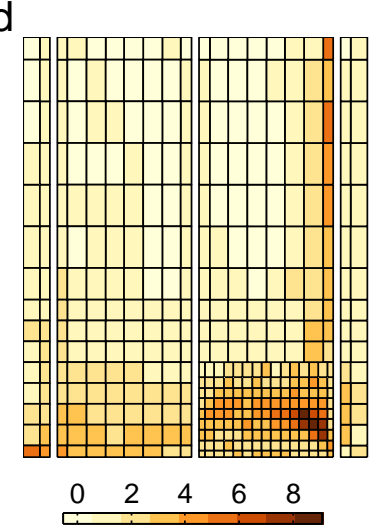

Figure 7: Maps of (a) mean, (b) standard deviation, (c) skewness and (d) excess of the pressure coefficients for a $340^{\circ}$ wind direction. 


\subsection{Description of the structure}

The roof is supported by 11 fixed-base frames placed every $5.72 \mathrm{~m}$. The second and third internal structural frames are used for illustrations and their positions are identified with triangles in Fig. 6, numbered from bottom to top in the figure.

Each frame is clamped and the connections are considered as infinitely rigid. Columns are HE 450 AA and beams are IPE 450 with a $235 \mathrm{MPa}$ steel grade and a $205000 \mathrm{MPa}$ Young's modulus. The finite element model of one frame, see Fig. 8, is an assembly of classical 2-D beam elements with three degrees of freedom per node (rotation, horizontal and vertical displacements).

Each frame is divided into 40 finite elements and the number of degrees of freedom is thus equal to 123 per frame. The aerodynamic pressure field acting on the cladding is transferred by the girts and purlins to each frame of the building (Main and Fritz, 2006). Girts and purlins, considered as hinged-hinged beams, are not modeled and each frame is analyzed separately. The design of the steel frames has been done following the Eurocode (2005).

Only two structural responses in the second and third frames, the bending moments at the nodes identified in Fig. 8 and labeled A and B, are considered to illustrate ESWLs. However, the bending moments at each node of the model are considered for the envelope reconstruction problem $\left(n_{r}=880\right)$ and the computation of the PSWLs. Envelope reconstruction using PSWLs is illustrated with both considered frames.

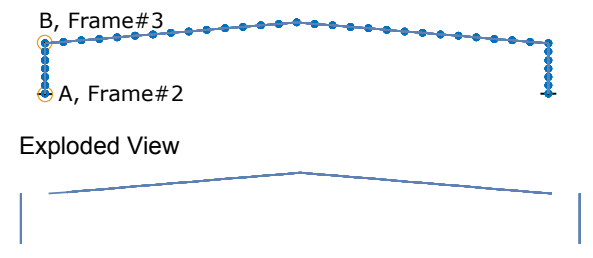

Figure 8: Elevation of a Frame. The dots identify the nodes of the finite element model. The bending moments at the two nodes A and B and identified by the circles are considered for the illustrations of ESWLs. The exploded view is used for the illustrations.

\subsection{Determination of the envelope}

Fig. 9 represents each step to compute the total envelope of the bending moments in both considered frames. For sake of clarity, only a scale is given for each graph and the numerical values for the two considered bending moments are reported in Tab. 1. 
Frame \#2
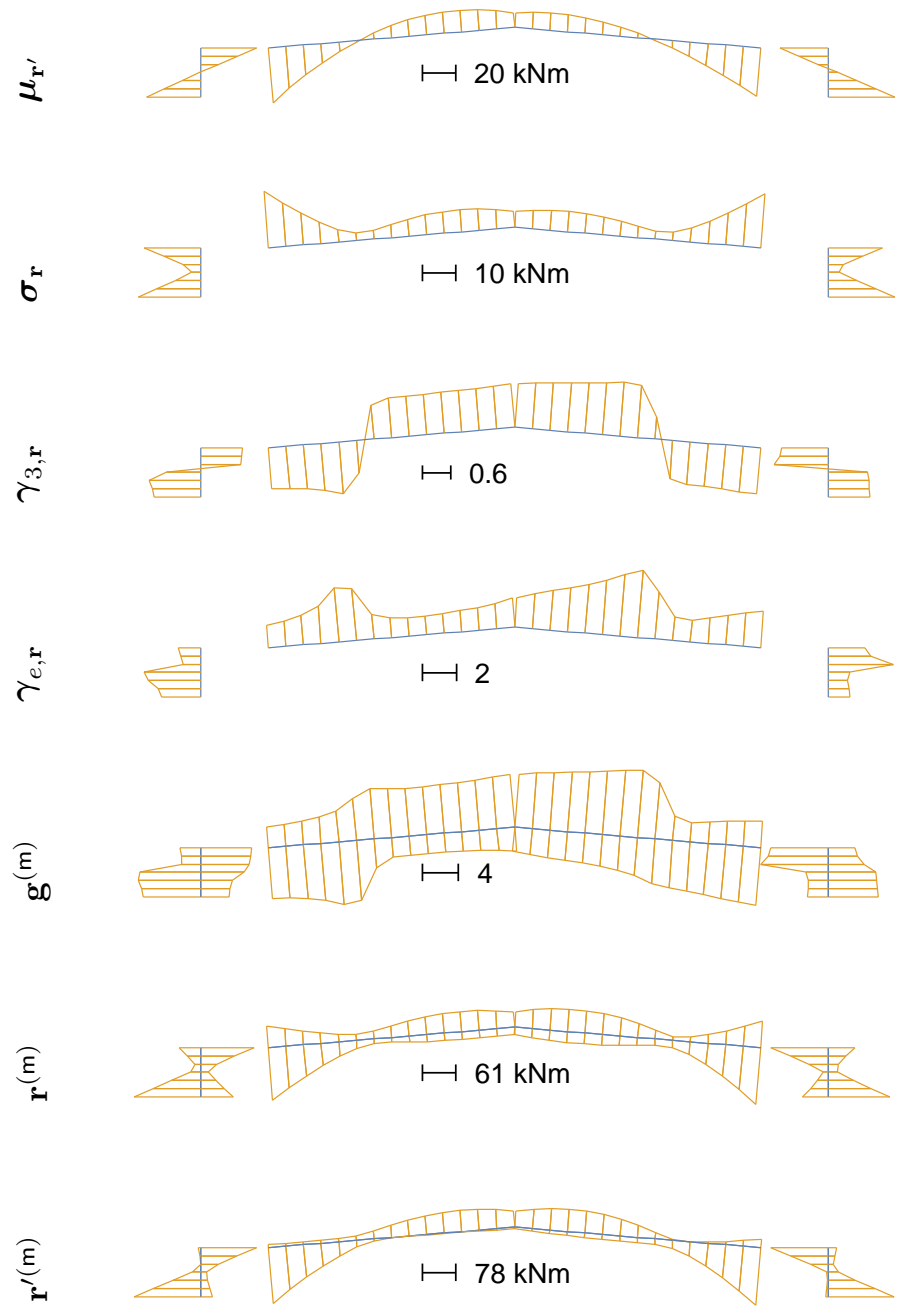

Frame \#3
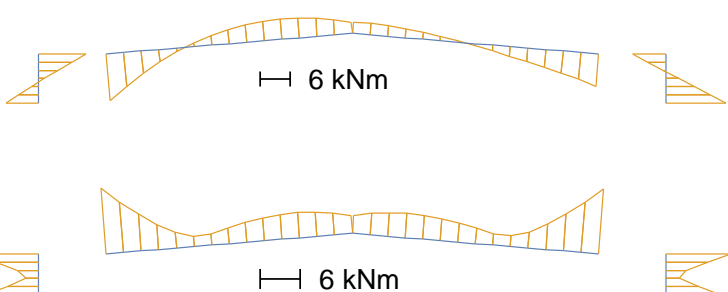

$\longmapsto 6 \mathrm{kNm}$
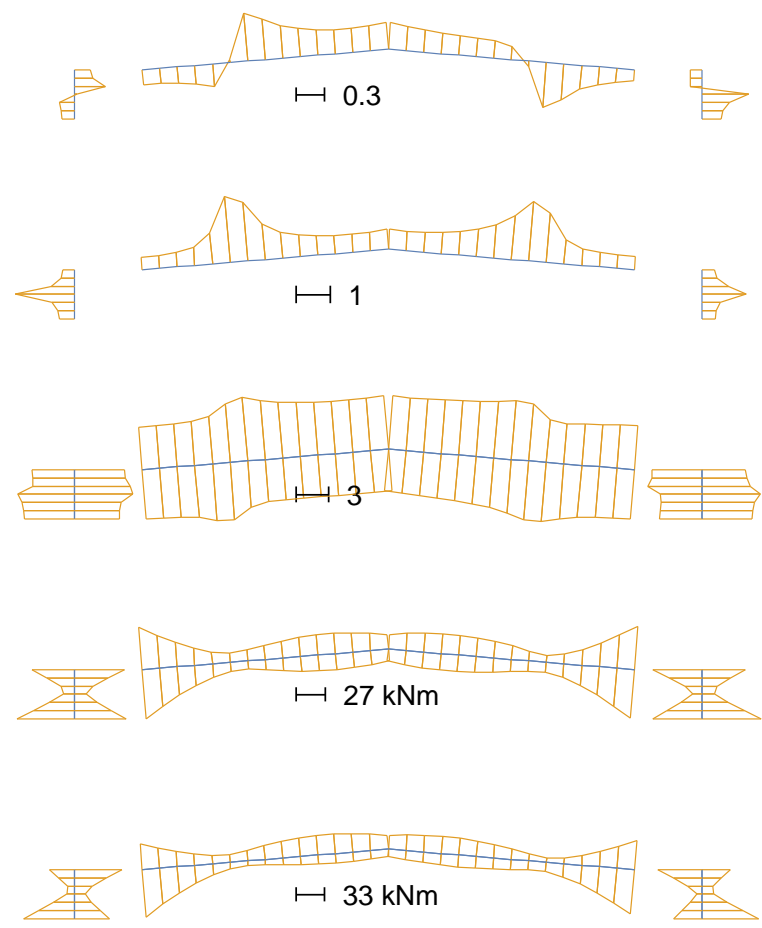

Figure 9: Mean, standard deviation, skewness coefficient, excess coefficient, peak factors, envelope and total envelope for the bending moments of both considered frames.

\begin{tabular}{|c|c|c|c|c|c|c|c|c|}
\cline { 2 - 9 } \multicolumn{1}{c|}{} & $\mu_{\mathrm{r}^{\prime}}$ & $\sigma_{\mathrm{r}}$ & $\gamma_{3, \mathrm{r}}$ & $\gamma_{e, \mathrm{r}}$ & $\mathrm{g}^{(\max )}$ & $\mathrm{g}^{(\min )}$ & $\mathrm{r}^{(\max )}$ & $\mathrm{r}^{(\min )}$ \\
\cline { 2 - 9 } \multicolumn{1}{c|}{} & {$[\mathrm{kNm}]$} & {$[\mathrm{kNm}]$} & {$[-]$} & {$[-]$} & {$[-]$} & {$[-]$} & {$[\mathrm{kNm}]$} & {$[\mathrm{kNm}]$} \\
\hline A (min), Frame \#2 & 32.8 & 19.0 & 1.0 & 2.3 & 6.4 & -3.0 & 121.5 & -57.9 \\
\hline B (max), Frame \#3 & -9.4 & 9.9 & -0.2 & 0.4 & 3.9 & -4.6 & 38.8 & -45.2 \\
\hline
\end{tabular}

Table 1: Numerical data associated with the two bending moments in Frame \#2 and Frame \#3, respectively.

The total envelope results from an element-by-element multiplication of the peak factors and the standard deviations and by adding the mean component. Bending moments in the second frame exhibit large skewness and excess coefficients resulting in an asymmetric envelope $\left(\mathbf{r}^{(\min )}, \mathbf{r}^{(\max )}\right)$. On the opposite, bending moments in the third frame exhibit moderate skewness and excess coefficients 
resulting in a nearly symmetric envelope, close to the limit case for Gaussian structural responses. Indeed, the aerodynamic pressure field acting on the third frame is nearly Gaussian.

Fig. 10 depicts the skewness and excess coefficients for the recorded aerodynamic pressures and the bending moments. Some processes are close to the monotone limitation and only a few (5 out of 1275 processes) are slightly outside. When necessary, the vertical mapping consisting in finding on the monotone limitation the skewness coefficient for the exact excess coefficient is applied, as recommended in (Peng et al., 2014).
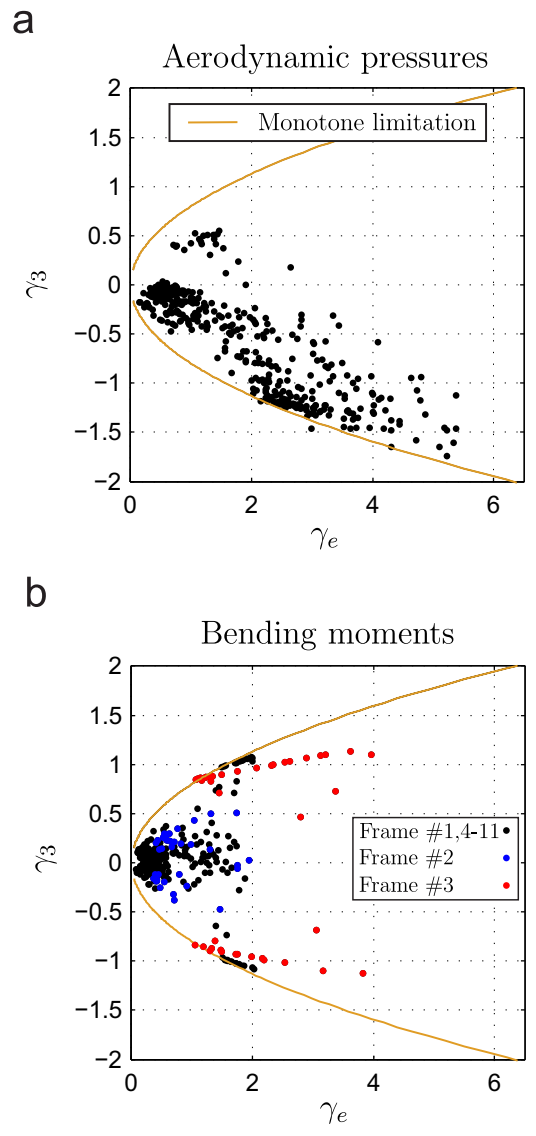

Figure 10: Skewness $\gamma_{3}$ and excess $\gamma_{e}$ coefficients for (a) aerodynamic pressures and for (b) bending moments.

\subsection{Equivalent static wind loads}

This section aims at comparing ESWLs computed with the 3 different methods described in Section 3: the Gaussian (LRC method), the non-Gaussian (CEL method with the bicubic model) and the conditional sampling technique. The magnitude of the coefficients, $\alpha^{(e, \mathrm{~m})}$ and $\boldsymbol{\beta}^{(e, \mathrm{~m})}$, applied to satisfy the envelope and non-overestimation conditions are compared to assess the formulation accuracy, i.e., those with the coefficients closest to unity are considered as more accurate. For the usual sampling technique, the load scaling coefficient $\alpha^{(e, \mathrm{~m})}$ has to be understood as the ratio between the envelope value and the one that would have been obtained from realisations. 
Fig. 11 illustrates the surfaces of influence, load-response correlation coefficients and responseresponse correlation coefficients for the two considered bending moments A (min) and B ( $\max )$. The load-response correlation and the response-response correlation coefficients help understanding the ESWL and structural response patterns, respectively. For example, the ESWL associated with response A produces the envelope value of the bending moment at the left support (Frame \#2); furthermore bending moments close to their envelope values are also expected in both frames at the connection between columns and beams and at mid-span where the correlation coefficients are large. This is confirmed in Fig. 13.

Response A, Frame \#2

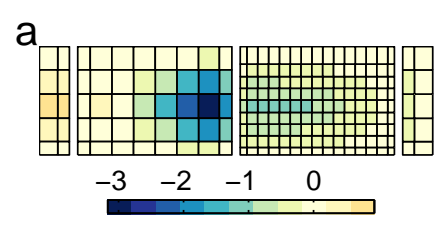

b
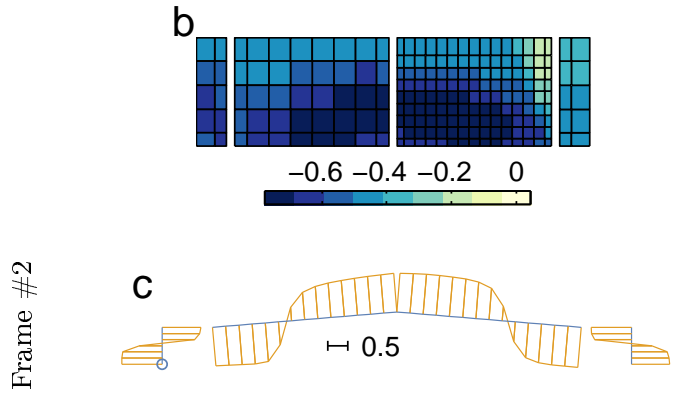

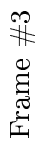

d

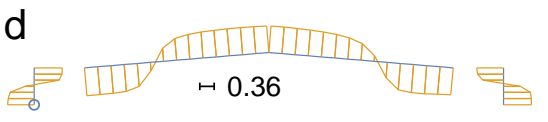

Response B, Frame \#3

a

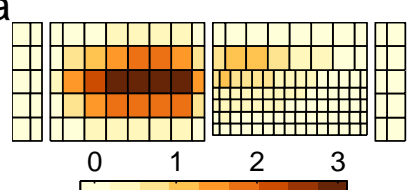

$\mathrm{b}$
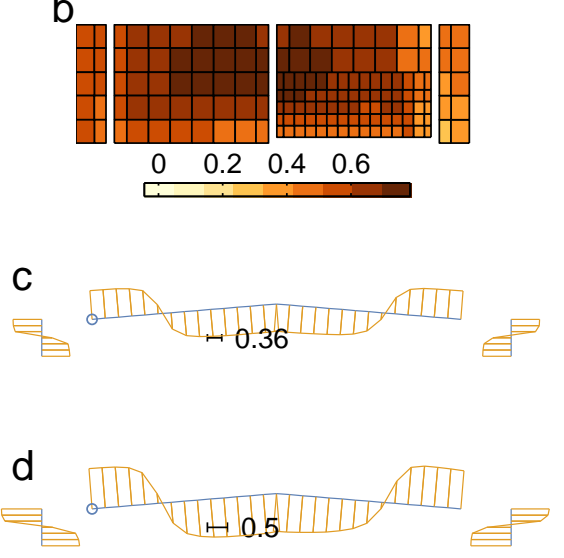

Figure 11: (a) Surface of influence, (b) load-response correlation coefficients and response-response correlation coefficients in (c) Frame \#2 and in (d) Frame \#3.

Fig. 12 illustrates the ESWLs associated with the mean largest minimum and maximum of the considered responses $\mathrm{A}$ and $\mathrm{B}$, respectively. First, third and fourth columns illustrate the original ESWLs, the coefficients $\boldsymbol{\beta}^{(e, \mathrm{~m})}$ and the adjusted ESWLs. Also the coefficients $\alpha^{(e, \mathrm{~m})}$ are given for each method. For response A, the three methods produce original (unadjusted) ESWLs which have similar patterns with slight differences in magnitude. This is not the case for the response B, for which the leading edge is less loaded with the ESWL computed from the sampling technique than with the two other methods. 
Bending moment $\mathbf{A}(\mathrm{m} \equiv \min )$

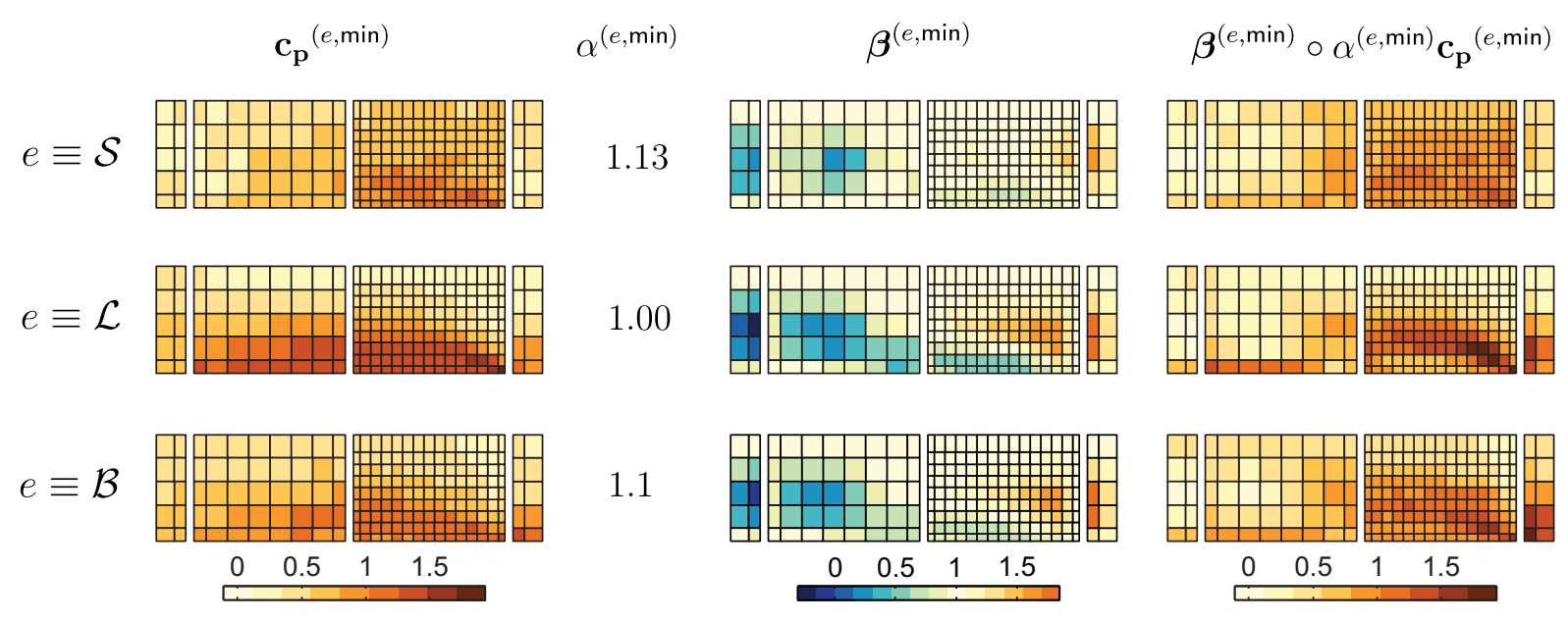

Bending moment $B(m \equiv \max )$

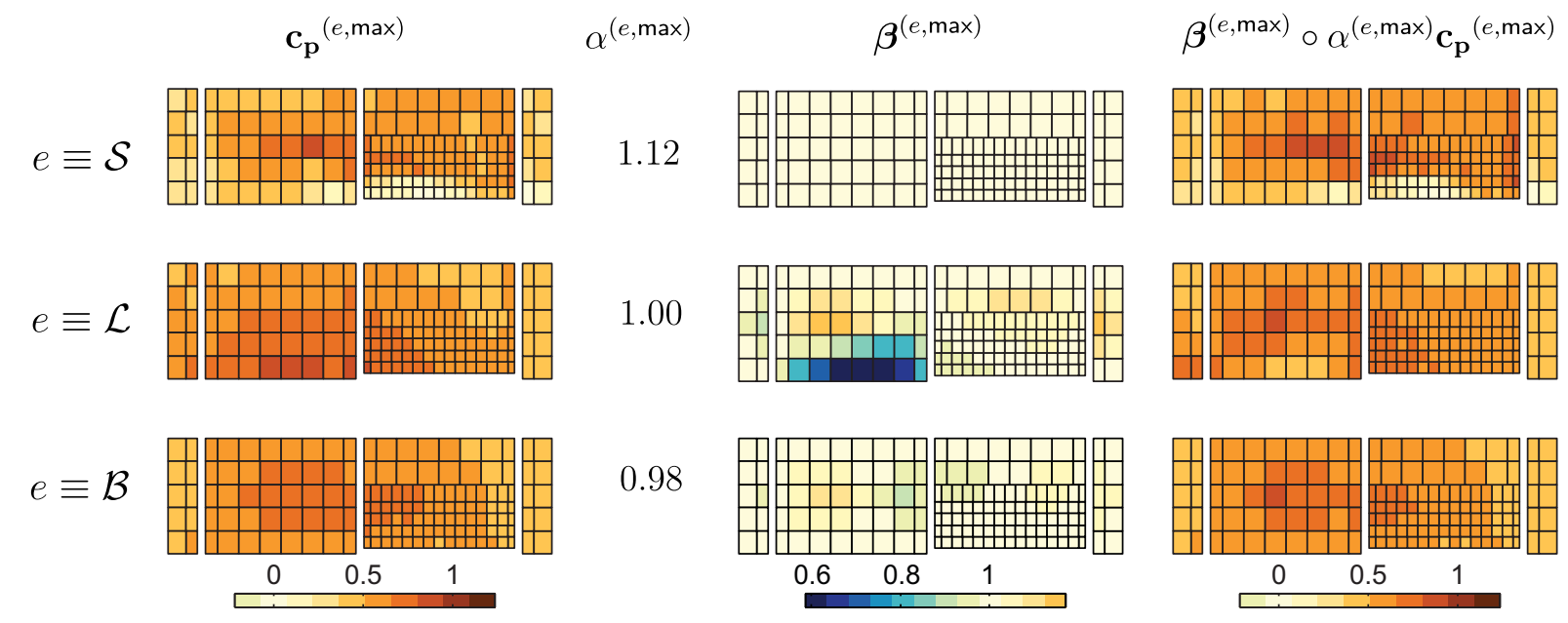

Figure 12: Original (left) and adjusted (right) ESWLs and coefficients $\alpha^{(e, m)}$ and $\boldsymbol{\beta}^{(e, \mathbf{m})}$ for the bending moments A $(\mathrm{m} \equiv \min )$ and $\mathrm{B}(\mathrm{m} \equiv \max )$. Conditional sampling technique $(e \equiv \mathcal{S})$, LRC method $(e \equiv \mathcal{L})$ and conditional expected load method with the bicubic model $(e \equiv \mathcal{B})$.

The target bending moment $\mathrm{A}$ is underestimated with the original ESWLs, i.e., the envelope value condition is satisfied with scaling coefficients larger than one, namely $\alpha^{(e, m i n)}=1.13$ for the sampling technique and $\alpha^{(e, \min )}=1.10$ for the non-Gaussian method. Nevertheless the target bending moment B is underestimated with the sampling technique $\left(\alpha^{(e, \max )}=1.12\right)$ and slightly overestimated $\left(\alpha^{(e, \max )}=0.98\right)$ with the non-Gaussian method.

The range of variation for coefficients $\boldsymbol{\beta}^{(e, \mathrm{~m})}$ is also larger for response A (up to 1.8) than for response B. For both responses, the coefficients $\boldsymbol{\beta}^{(e, m)}$ obtained with the sampling technique and the non-Gaussian method are lower than those necessary for the LRC method. For response B, the 
coefficients $\boldsymbol{\beta}^{(e, \mathrm{~m})}$ obtained with the sampling technique and the non-Gaussian method are close to one, i.e., the non-overestimation condition is nearly fulfilled under the scaled ESWL; slight adjustments are sufficient. Conversely for the LRC method, a larger range of variation for the coefficients $\boldsymbol{\beta}^{(e, m)}$ is necessary.

Figures 13-14 illustrate the static bending moments in the structure under the two scaled ESWLs associated with the bending moments $\mathrm{A}$ and $\mathrm{B}$, respectively. The bending moments under the original and the adjusted ESWLs provide very similar profiles. The responses under the three kinds of loadings (original, scaled and adjusted) are distinguished with a discrepancy indicator $\varepsilon^{(e)}$ that measures the relative differences between the envelope $\left(\mathbf{r}^{(\max )}, \mathbf{r}^{(\min )}\right)$ and the responses $\mathbf{r}^{(e, \mathrm{~m})}$ under the ESWLs, such as

$$
\boldsymbol{\varepsilon}^{(e)}=\max \left\{\left(\mathbf{r}^{(e, \mathrm{~m})}-\mathbf{r}^{(\min )}\right) \div \mathbf{r}^{(\min )},\left(\mathbf{r}^{(e, \mathrm{~m})}-\mathbf{r}^{(\max )}\right) \div \mathbf{r}^{(\max )}\right\}
$$

where division is performed element by element. Three such indicators exist and are represented in Figs. 13 and 14. 
Bending moment $\mathbf{A}(\mathrm{m} \equiv \min )$

Frame \#2

Frame \#3

Conditional sampling technique $(e \equiv \mathcal{S})$

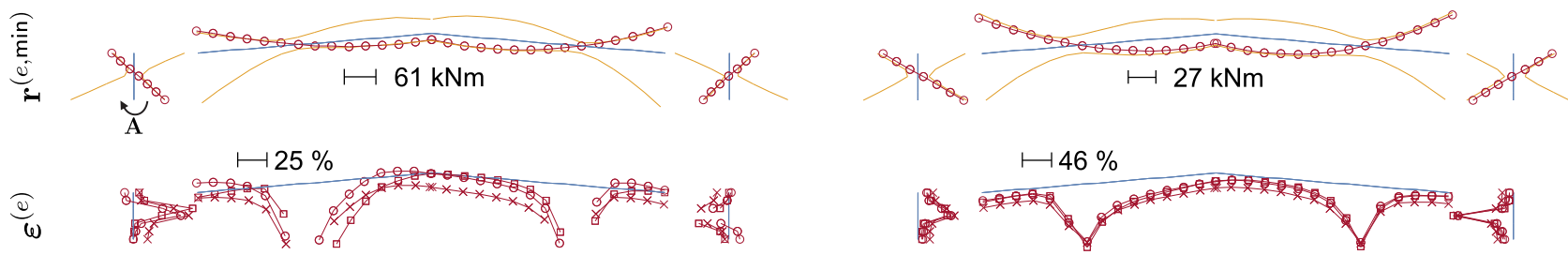

Load-response correlation method $(e \equiv \mathcal{L})$
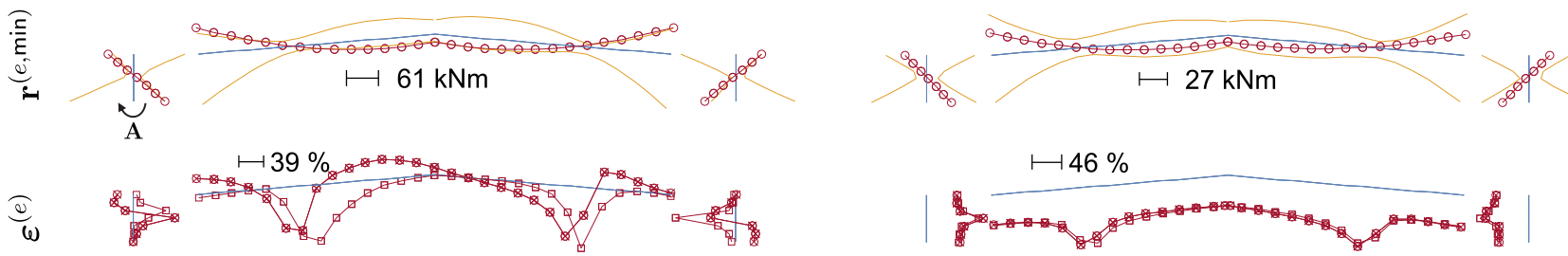

Conditional expected load method $(e \equiv \mathcal{B})$

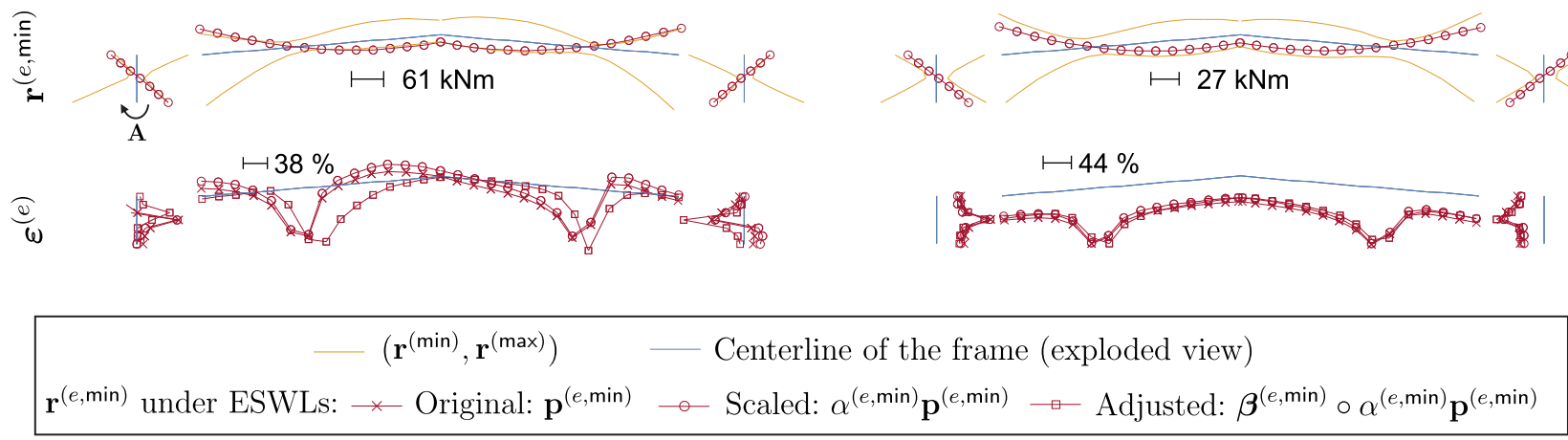

Figure 13: Static bending moments $\mathbf{r}^{(e, \min )}$ under scaled ESWLs associated with bending moment A. Relative differences $\varepsilon^{(e)}$ between the envelope of the bending moments and the bending moments obtained under the original, the scaled and the adjusted ESWLs. Positive relative differences (overestimations) are depicted outside the frame. Large (unimportant) negative relative differences (depicted inside the frame) are not shown for sake of clarity.

For the bending moment A (in Frame \#2), after scaling and before adjustment of the ESWL (Fig. 13, o markers), overestimations do not occur in sections adjacent to the considered bending moment but in other parts of Frame \#2, where the correlation with the bending moment A is large. No overestimation occurs in Frame \#3 when considering the ESWLs associated with bending moment A. For the bending moment B (in Frame \#3), after scaling and before adjustment of the ESWL (Fig. 14, o markers), largest overestimations occur with the LRC method, hence the highest range of variation for the coefficients $\boldsymbol{\beta}^{(e, \max )}$. With the LRC method, no overestimation occurs in Frame \#3 but very large overestimations (nearly 40\%) occur in Frame \#2. These overestimations take place 
Bending moment $B(m \equiv \max )$

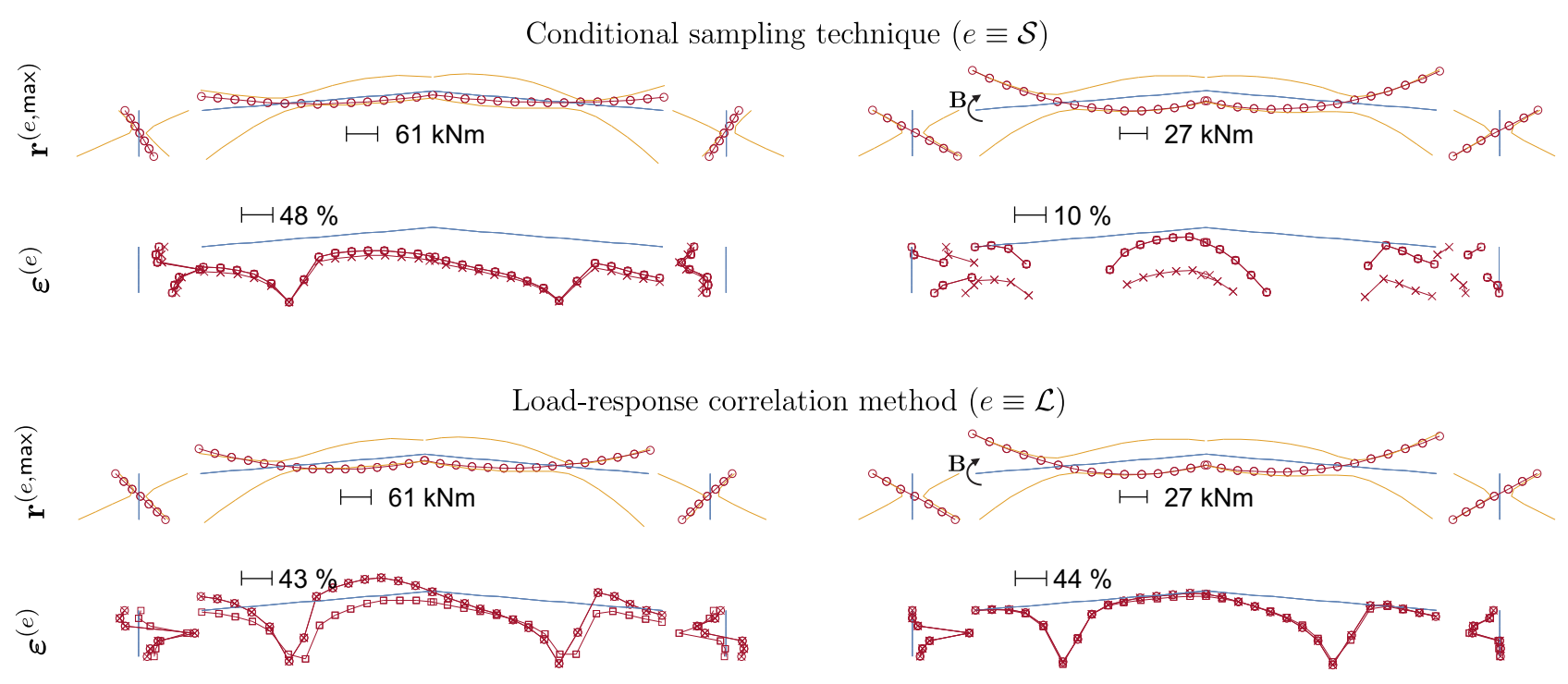

Conditional expected load method $(e \equiv \mathcal{B})$

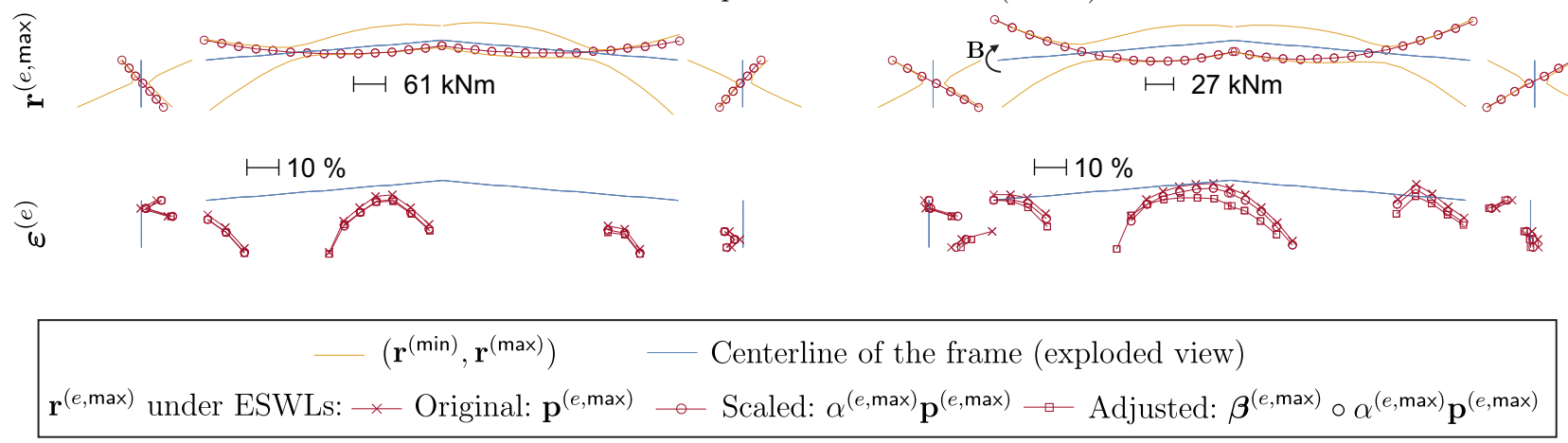

Figure 14: Static bending moments $\mathbf{r}^{(e, \max )}$ under scaled ESWLs associated with bending moment B. Relative differences $\varepsilon^{(e)}$ between the envelope of the bending moments and the bending moments obtained under the original, the scaled and the adjusted ESWLs. Positive relative differences (overestimations) are depicted inside the frame. Large (unimportant) negative relative differences (depicted inside the frame) are not shown for sake of clarity. 
where $(i)$ correlation with the bending moment B is large, Fig. 11, and (ii) peak factors, Fig. 9, are very different, as demonstrated by Eq. (13).

We have also computed the ESWLs for each bending moment (in Frames \#2 and \#3) and for both envelopes (min and max). Fig. 15 illustrates the associated scaling coefficients $\alpha^{(e, m)}$ and also maximum of the relative differences $\varepsilon^{(e)}$ to the envelope of the static responses under the scaled ESWLs. Similar range of variation for the scaling coefficients $\alpha^{(e, m)}$ are observed for the sampling technique and the non-Gaussian method. For the sampling technique, the fact that the envelope value condition is not fulfilled is due to the low number of 10-min observation windows. Indeed, provided the peak factor used for the envelope is the exact one no scaling coefficient would be needed. We also recall that the scaling coefficients $\alpha^{(e, m)}$ are exactly equal to unity for the LRC method. In terms of overestimation of the envelopes $\mathbf{r}^{(e, m)}$ under the scaled ESWLs, it is observed that $(i)$ in Frame \#2, larger overestimations are observed (up to 60\%) with the LRC method (cross markers) than with the two other methods and $(i i)$ in Frame \#3, large overestimations are only observed with the LRC method while the other two methods perform very well. Note that the sampling technique performs slightly better than the non-Gaussian method, especially for the bending moments in Frame $\# 2$.

Frame \#2
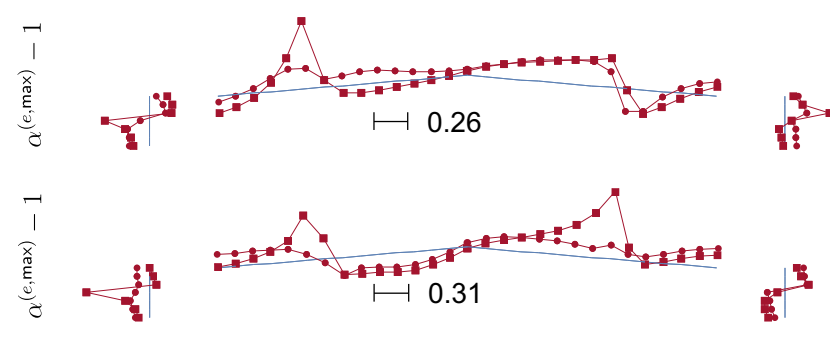

$$
\alpha^{(e, m a x)} \mathbf{p}^{(e, m a x)}
$$
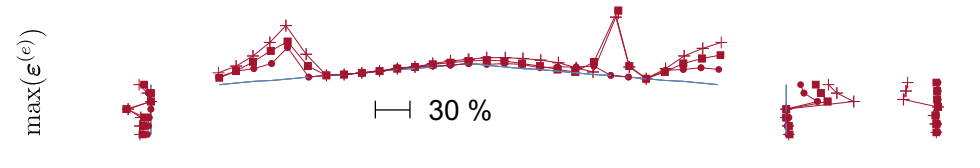

$$
\alpha^{(e, \min )} \mathbf{p}^{(e, \min )}
$$

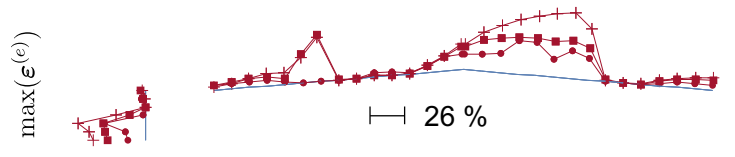

Frame \#3
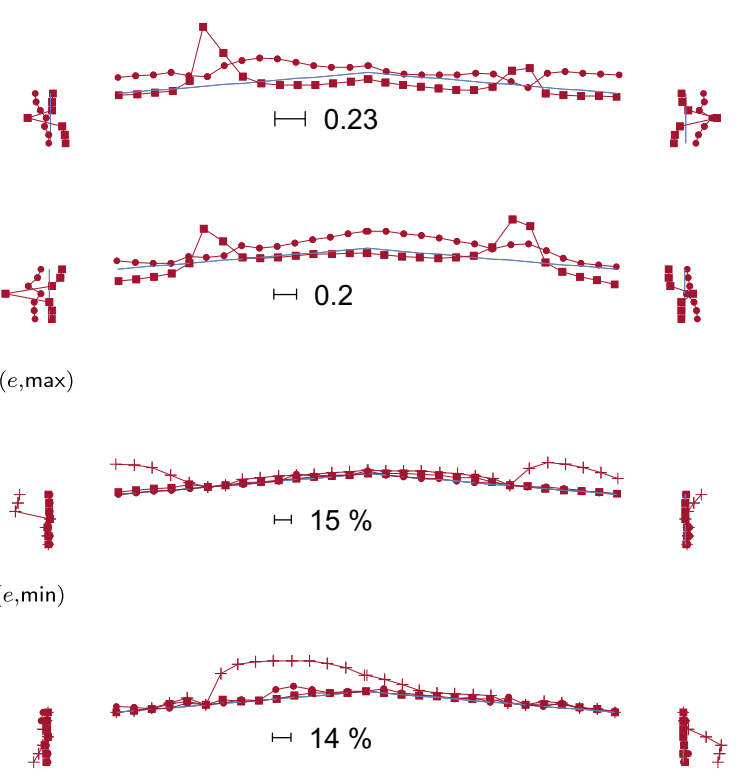

Results obtained with ESWLs: $\bullet$ CST-based $(e \equiv \mathcal{S})+$ LRC-based $(e \equiv \mathcal{L}) \bullet$ CEL-based (bicubic model $)(e \equiv \mathcal{B})$

Figure 15: Coefficients $\alpha^{(e, \mathrm{~m})}$ that scale the original ESWLs computed for both envelope values for each bending moment in Frame \#2 and Frame \#3. Maximum of the relative differences $\varepsilon^{(e)}$ to the envelope of the static responses under scaled ESWLs computed for each bending moment in Frame \#2 and Frame \#3.

To summarize, the non-overestimation condition seems to be more easily fulfilled with the ESWLs derived from the sampling technique and the non-Gaussian method than with the LRC method. The 
extension of the original LRC method to a non-Gaussian formalism improves the fulfillment of the non-overestimation condition, i.e., smaller coefficients $\boldsymbol{\beta}^{(e, m)}$ are necessary than those obtained with the LRC method.

In the light of these results, the use of ESWLs with non-Gaussian structural responses should receive more attention and we should opt for the ESWLs derived from the sampling technique or the proposed non-Gaussian method instead of the LRC method. An additional reason why we advise the use of PSWLs for the ERP: the problem of overestimation of the envelope does not occur.

\subsection{Principal static wind loads}

This section assesses the envelope reconstruction efficiency of the bending moments in the eleven frames using combinations of a limited number of PSWLs. The matrix $\mathbf{P}^{(p)}$ collecting PSWLs results from the singular value decomposition of $\mathbf{P}^{(e)}$, the $395 \times 1760\left(n_{l} \times 2 n_{r}\right)$ matrix collecting all ESWLs obtained either by the conditional sampling technique (CST-based PSWLs) or the LRC method (LRC-based PSWLs) or the CEL method with the bicubic model (CEL-based PSWLs). This matrix factorization is straightforward and the PSWLs are obtained right away. The normalized cumulative summation of the principal coordinates $\mathrm{S}_{i i}$ is shown in Fig. 16.

The LRC method has the largest principal coordinates while the sampling technique has the smallest. The ESWL basis obtained with the sampling technique are actually more dissimilar than in the two other approaches. The ESWLs are sampled from pressure field and the small number of 10-min observation windows makes it such that there is more variability in the ESWLs associated with different responses, than what the LRC or CEL approaches - based on smooth models - provide.

PSWLs have to be first normalized before using them for the envelope reconstruction problem and Fig. 17 illustrates the first four normalized PSWLs.

The first three principal loadings show important similarities in their pattern and magnitude for the three methods while the fourth principal loading obtained with the sampling technique is completely different. The first principal loading produces a suction on the entire roof while the second and third exhibit asymmetric patterns, between the windward edge and the roof behind and between the western and eastern parts of the roof, respectively. The second normalization for any PSWL is obtained with

$$
\mathbf{C}_{\mathbf{p}, i}^{(p, 2)}=-\frac{\alpha_{i}^{(p, 2)}}{\alpha_{i}^{(p, 1)}} \mathbf{C}_{\mathbf{p}, i}^{(p, 1)}
$$

see Eq. (37). Note that the ratio $\alpha_{i}^{(p, 2)} / \alpha_{i}^{(p, 1)}$, given in Fig. 17, has a large range of variation [0.49 1.79] here while it is equal to one in a Gaussian framework.

Fig. 18 illustrates the static bending moments under $\mathbf{C}_{\mathbf{p}, 1}^{(p, 1)}, \mathbf{C}_{\mathbf{p}, 1}^{(p, 2)}, \mathbf{C}_{\mathbf{p}, 3}^{(p, 1)}$ and $\mathbf{C}_{\mathbf{p}, 3}^{(p, 2)}$ in Frames \#2 and \#3 as well as relative differences between the static responses $\mathbf{R}_{i}^{(p, 1)}$ and the envelope, defined as

$$
\varepsilon_{i}^{(p, 1)}=\max \left\{\left(\mathbf{R}_{i}^{(p, 1)}-\mathbf{r}^{(\min )}\right) \div \mathbf{r}^{(\min )},\left(\mathbf{R}_{i}^{(p, 1)}-\mathbf{r}^{(\max )}\right) \div \mathbf{r}^{(\max )}\right\} .
$$




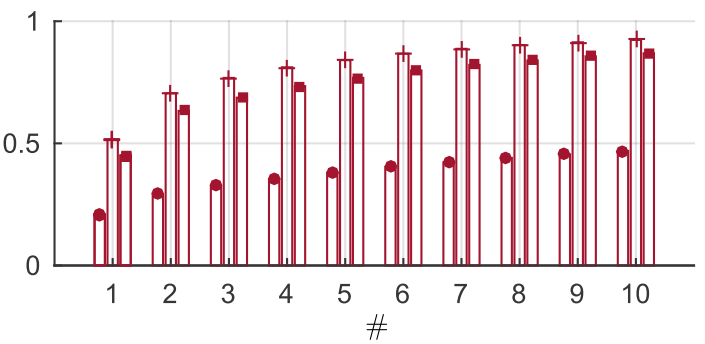

PSWLs: $\bullet$ CST-based +LRC-based $\bullet$ CEL-based (bicubic model)

Figure 16: Normalized cumulative summation of the principal coordinates of the principal loadings. 

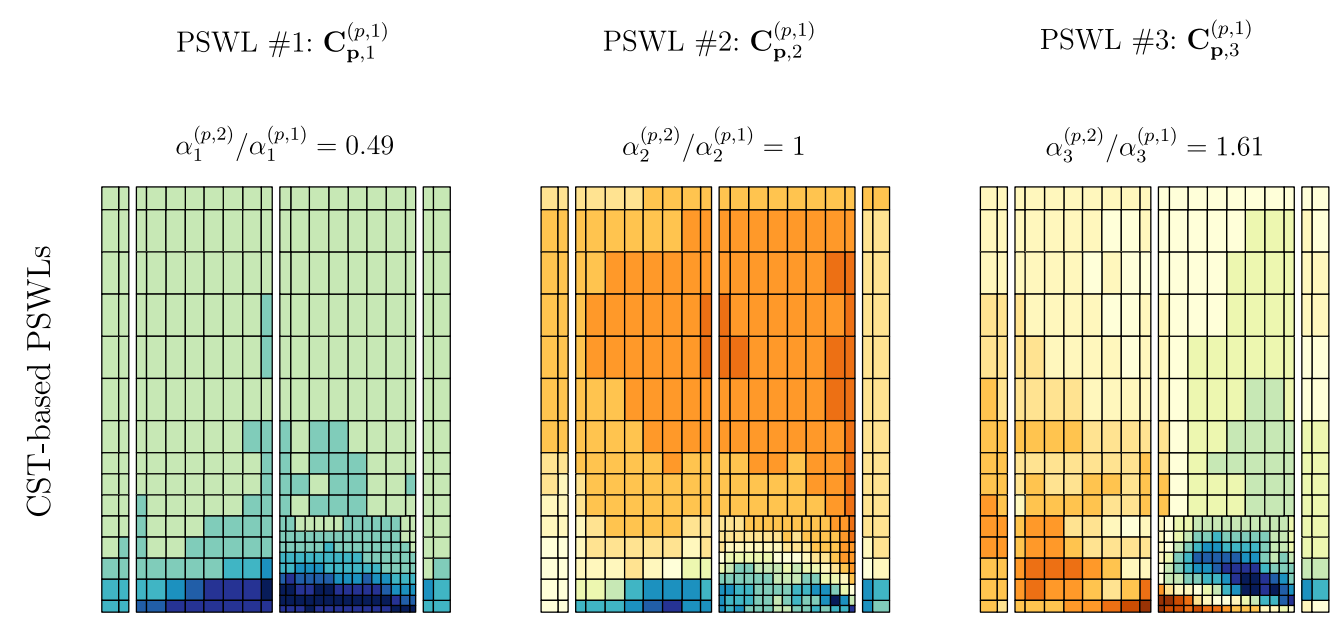

PSWL \#4: $\left.\mathbf{C}_{\mathbf{p}, 4}^{(p, 1)}\right)$
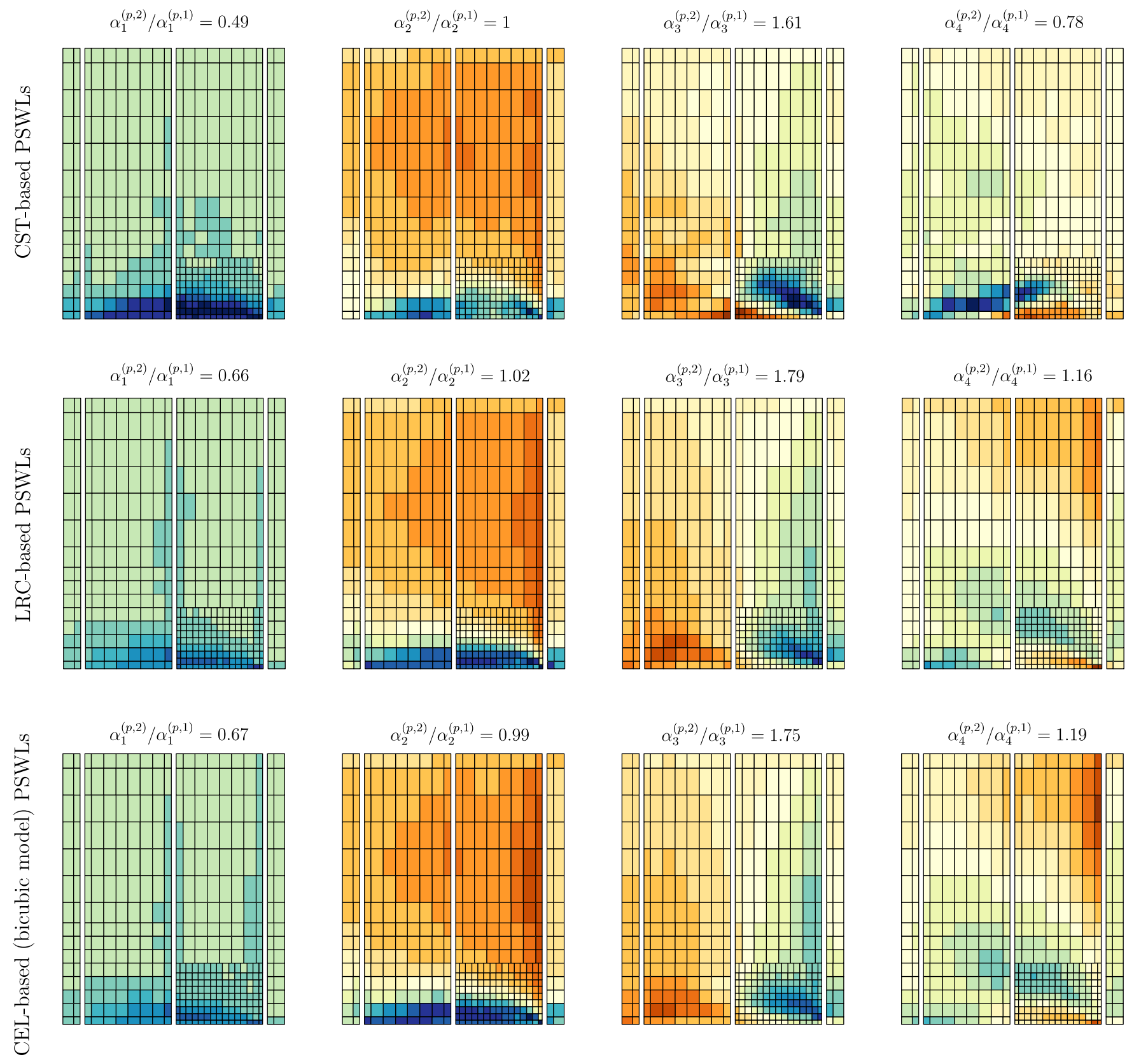

Range of aerodynamic coefficients for each column

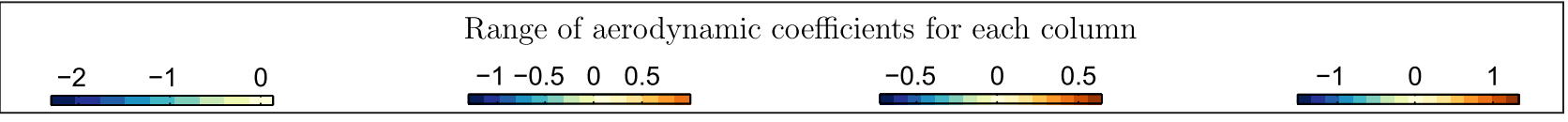

Figure 17: First four normalized PSWLs. 
Frame \#2
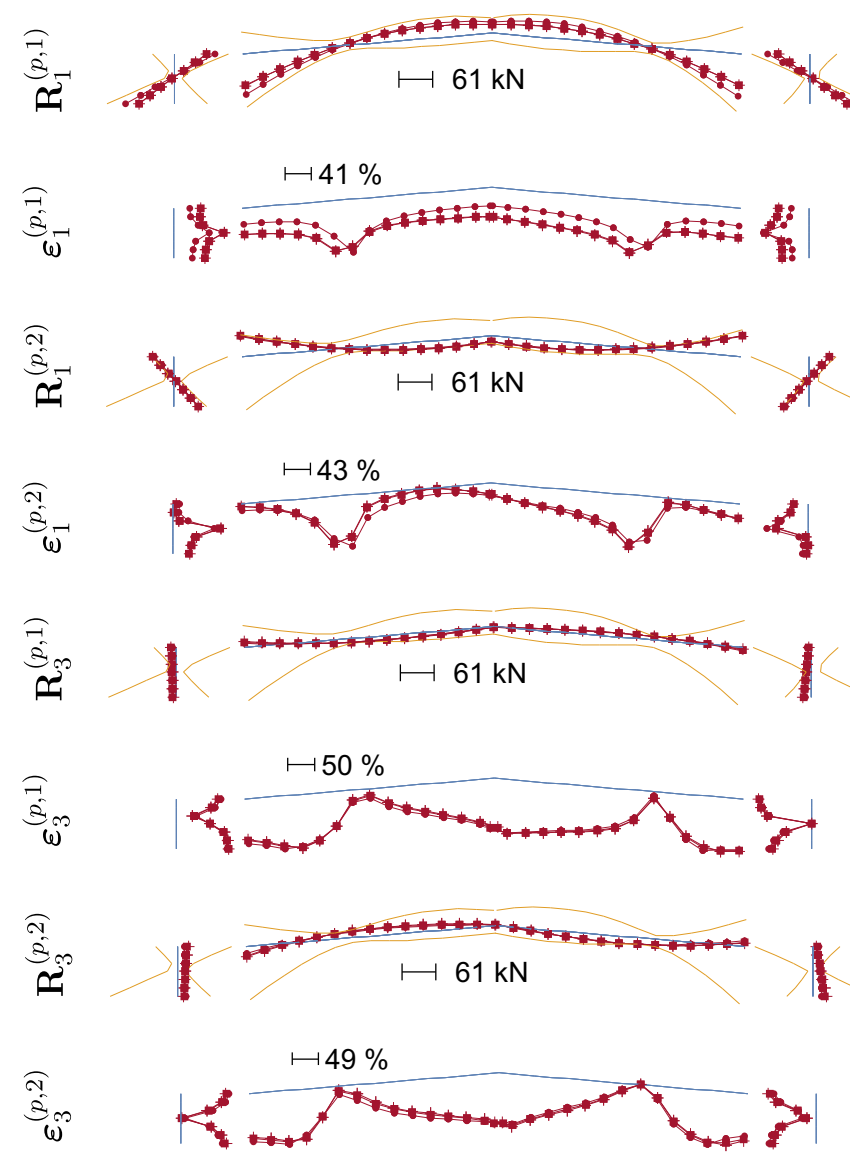

Frame \#3
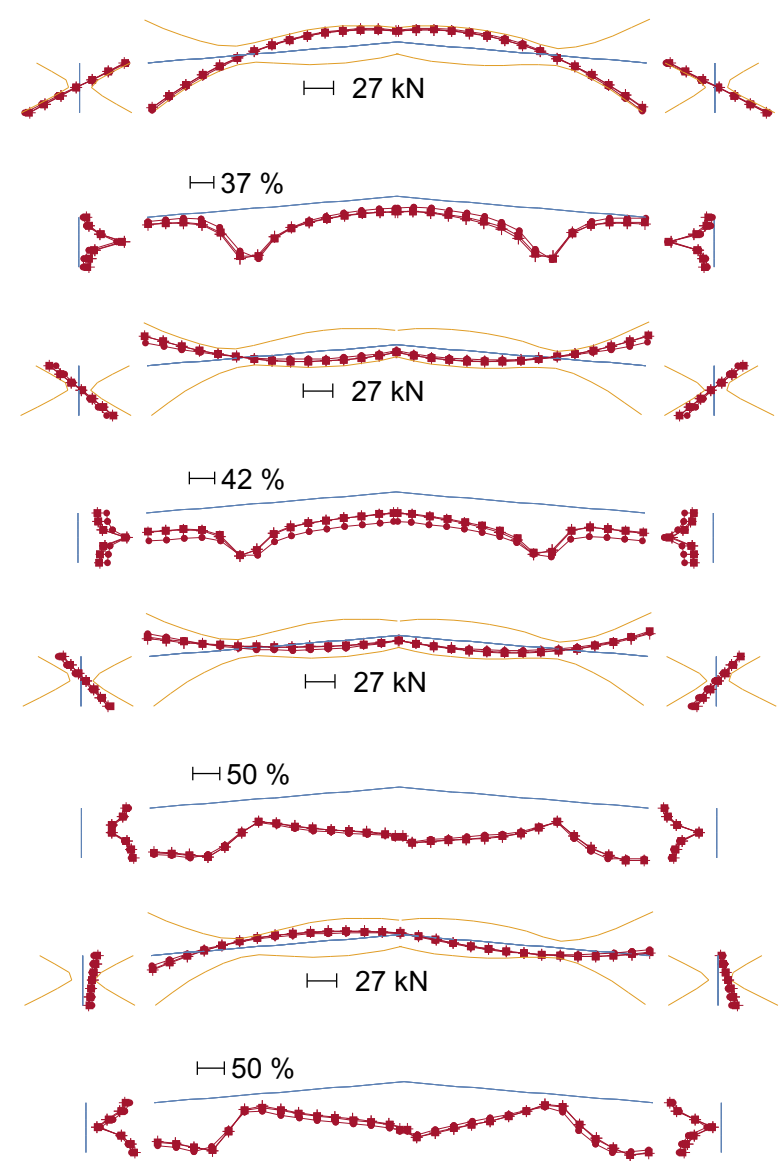

Results obtained under PSWLs: • CST-based +LRC-based • CEL-based (bicubic model)

Figure 18: Static bending moments and relative differences with the envelope under $\mathbf{C}_{\mathbf{p}, 1}^{(p, 1)}, \mathbf{C}_{\mathbf{p}, 1}^{(p, 2)}, \mathbf{C}_{\mathbf{p}, 3}^{(p, 1)}$ and $\mathbf{C}_{\mathbf{p}, 3}^{(p, 2)}$ in Frames \#2 and \#3.

Bending moments under the PSWLs computed with the LRC method and the non-Gaussian method are almost similar while disparities are observed with the bending moments under $\mathbf{C}_{\mathbf{p}, 1}^{(p, 1)}$ and $\mathbf{C}_{\mathbf{p}, 1}^{(p, 2)}$ computed with the sampling technique. For the sampling technique, lower relative differences are observed under $\mathbf{C}_{\mathbf{p}, 1}^{(p, 1)}$ in comparison with the two other methods, but larger relative differences are observed under $\mathbf{C}_{\mathbf{p}, 1}^{(p, 2)}$. Notice that the PSWLs $\mathbf{C}_{\mathbf{p}, 3}^{(p, 1)}$ and $\mathbf{C}_{\mathbf{p}, 3}^{(p, 2)}$ produce large bending moments at the specific sections where the bending moments under $\mathbf{C}_{\mathbf{p}, 1}^{(p, 1)}$ and $\mathbf{C}_{\mathbf{p}, 1}^{(p, 2)}$ are rather low, see at mid-height of the columns and at quarter-span and three quarter-span of the roof.

Fig. 19 illustrates the reconstructed envelope with ten load cases: $(i)$ the first five PSWLs along with both normalizations are applied successively (no combinations) and ( $i i$ ) with optimized combinations of the first four PSWLs. As expected, the reconstructed envelopes in both frames have the same 
range of relative errors, since PSWLs and combinations thereof aim at a global reconstruction of the bending moments in the whole structure (eleven frames). The reconstructed envelope is not satisfactory applying the first 5 PSWLs without combination: relative errors are large, up to $-65 \%$. For the same number of load cases, ten combinations of the first 4 PSWLs produce a satisfactory reconstruction of the envelope; the largest relative errors are observed in sections where the bending moments are low.

$$
\text { Frame \#2 Frame \#3 }
$$

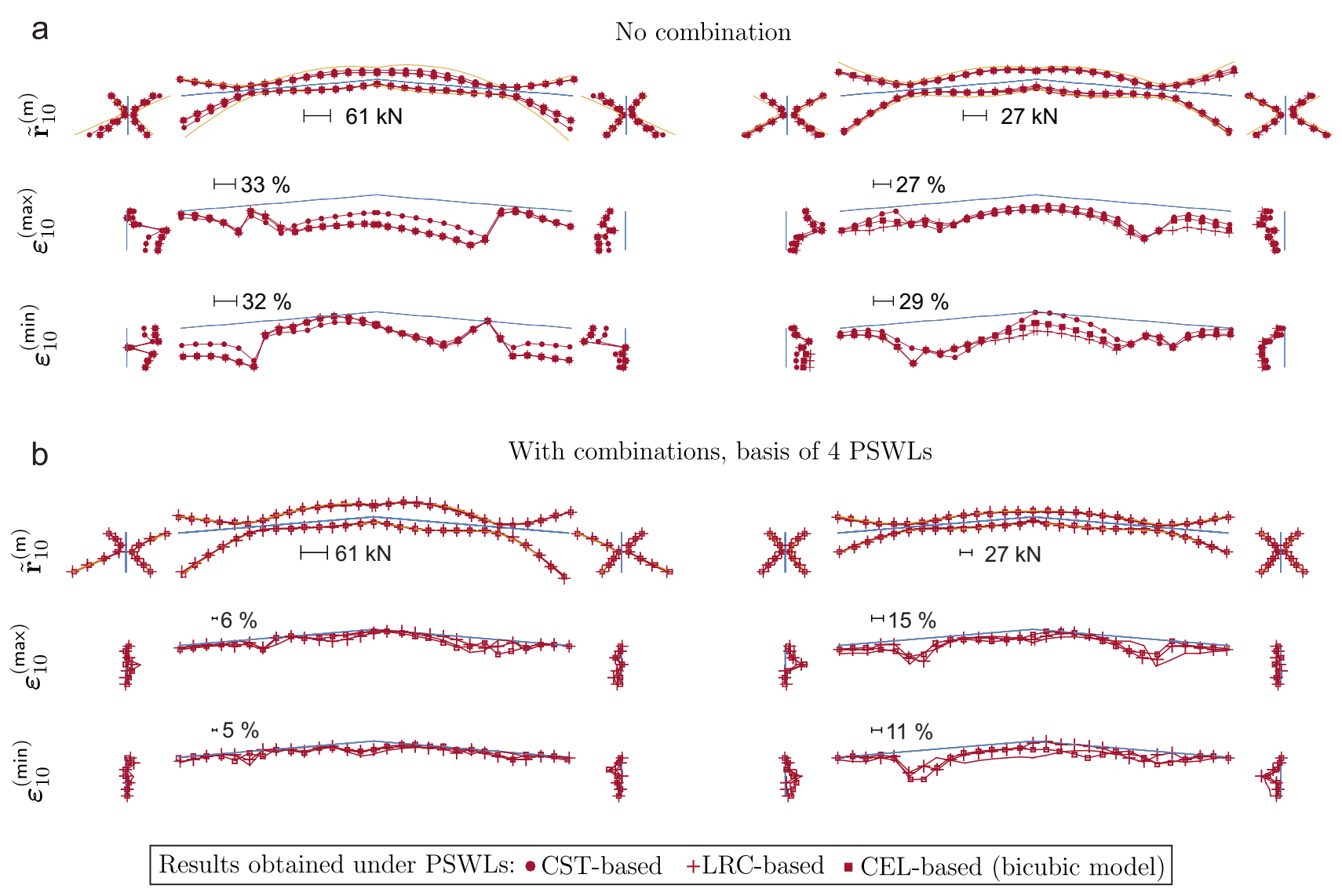

Figure 19: Reconstructed envelope and associated relative errors with ten load cases.

The overall envelope reconstruction accuracy is assessed by computing first two indicators

$$
\mathcal{R}_{(k)}^{(\mathrm{m})}=\mathbb{E}\left[\tilde{\mathbf{r}}_{(k)}^{(\mathrm{m})} \div \mathbf{r}^{(\mathrm{m})}\right]
$$

chosen here as the percentage of reconstruction for each side of the envelope in average and next

$$
\mathcal{R}_{(k)}=\frac{\mathcal{R}_{(k)}^{(\min )}+\mathcal{R}_{(k)}^{(\max )}}{2},
$$

that is called the envelope reconstruction indicator. 
The indicator of reconstruction $\mathcal{R}_{(k)}$ gives a global picture of the whole reconstruction of the bending moments in the entire structure, i.e., in all eleven frames. The evolution of $\mathcal{R}_{(k)}$ is depicted as a function of the number of load cases (from 2 to 10) derived by successive applications of PSWLs (no combinations), or combinations of them (two, four and eight PSWLs are combined), see Fig. 20. The evolution of $\mathcal{R}_{(k)}$ features a slow monotonic increase.
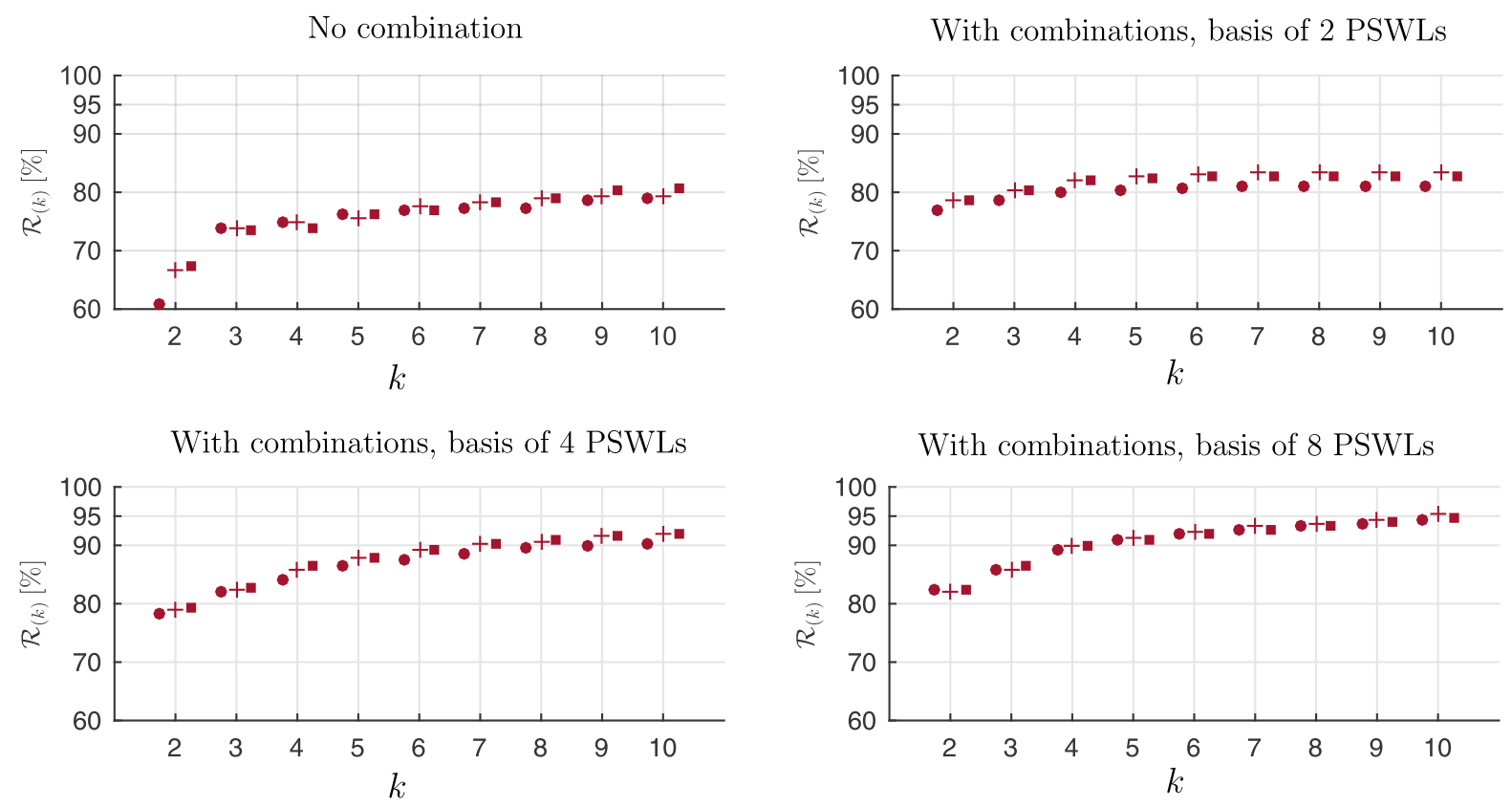

Results obtained by PSWLs: • CST-based +LRC-based - CEL-based (bicubic model)

Figure 20: Evolution of the envelope reconstruction indicator $\mathcal{R}_{(k)}$ as a function of the number of load cases.

The reconstruction obtained with the conditional sampling technique performs slightly worse than with the two other approaches but this is not significant. Applying PSWLs without combination gives a value of $\mathcal{R}_{(k)}$ around $80 \%$ for 10 load cases and 10 combinations of the first 2 PSWLs do not bring a significant improvement. However, combinations of the first 4 and 8 PSWLs gives a value of $\mathcal{R}_{(k)}$ around $90 \%$ and $95 \%$, respectively. The compromise is to find a minimum number of load cases required to achieve a fixed-level for $\mathcal{R}_{(k)}$ by minimizing the number $n_{p}$ of PSWLs used for combinations. If the number of load cases $n_{c}$ or the number $n_{p}$ of PSWLs used for combinations to consider is too large, one should re-consider another value for $\mathcal{R}_{(k)}$ or relax the tangency condition, i.e., accept overestimations of the envelope in order to achieve a minimum level for $\mathcal{R}_{(k)}$.

\section{Summary}

Equivalent static wind loads have been derived and directly used as a valuable tool in the design of structures. Further researches put the concept forward by deriving static wind loads that are no longer associated with one specific structural response, to tackle more efficiently the envelope reconstruction 
problem. In a Gaussian framework, the PSWL concept has been recently introduced as an optimum basis for this purpose.

Since aerodynamic pressures may exhibit mildly to strongly non-Gaussianities and therefore produce non-Gaussian structural responses, we have extended the method of PSWLs to a non-Gaussian framework. Substantial modifications to the original formulation are made to deal with asymmetric envelopes of structural responses. A methodology to combine efficiently several PSWLs is proposed using constrained nonlinear optimization. It has been demonstrated that PSWLs are still well-suited to form a reduced basis of loadings and, by combination, produce an accurate reconstruction of asymmetric envelopes.

PSWLs are derived from an SVD operation of ESWLs, hence a novel formulation of ESWL for structures with quasi-static behavior and subjected to non-Gaussian aerodynamic pressures has been studied. Inspired by the LRC method in which Gaussian conditional probability densities as well as their mean values are required, the concept of conditional expected static wind load is introduced. It is defined as the average wind loads conditioned upon recovery of the considered response and a novel non-Gaussian formulation of an ESWL has been obtained from a bicubic model of non-Gaussian conditional probability density. We have shown that this approach regularly extends the well-know LRC method. Two other methods have been considered for comparison: the conditional sampling pressure technique and the original LRC method.

In order to compare those methods, two required properties of an ESWL have been formulated and investigated: the envelope value and non-overestimation conditions. Indeed, the studied ESWL formulations may not naturally satisfy these two conditions and a procedure is proposed to scale and adjust original ESWLs whenever necessary. It has been illustrated that the LRC method may encounter some difficulties to satisfy the non-overestimation condition, i.e., large local coefficients have to be applied to the original ESWLs. Actually, the conditional sampling technique and the proposed non-Gaussian method are better suited: they satisfy the non-overestimation condition without excessively distorting original ESWLs, i.e., adjusted ESWLs remain close to the original ones. Computing ESWLs and using them as such, we thus recommend the use of the proposed conditional expected load method or the sampling technique instead of the LRC method in case of non-Gaussian structural responses.

However, concerning the envelope reconstruction efficiency, no significant differences on the reconstruction efficiency are observed between PSWLs based on the ESWLs obtained with any of the three investigated methods.

\section{Acknowledgments}

Luca Caracoglia is acknowledged for recommending us the NIST aerodynamic database for the illustration of our study. Also, the reviewers are thanked for their critical comments and suggestions to improve the present paper.

\section{References}

Atta, C., 1974. Sampling techniques in turbulence measurements. Annual Review of Fluid Mechanics $6,75-91$. 
Blaise, N., Denoël, V., 2013. Principal static wind loads. Journal of Wind Engineering and Industrial Aerodynamics 113, 29-39.

Blaise, N., Hamra, L., Denoël, V., 2012. Principal static wind loads on a large roof structure, in: Proceedings of the 12th ANIV conference of wind engineering In Vento.

Chen, X.Z., Kareem, A., 2001. Equivalent static wind loads for buffeting response of bridges. Journal of Structural Engineering-Asce 127, 1467-1475.

Choi, M., Sweetman, B., 2010. The hermite moment model for highly skewed response with application to tension leg platforms. Journal of Offshore Mechanics and Arctic Engineering 132, 021602.

Davenport, A.G., 1964. Note on the distribution of the largest value of a random function with application to gust loading, in: ICE Proceedings, Thomas Telford. pp. 187-196.

Denoël, V., Maquoi, R., 2012. The concept of numerical admittance. Archive of Applied Mechanics 82, 1337-1354.

Eurocode, E., 2005. EN 1993-1-1: Eurocode 3: Design of Steel Structures. Part 1-1: General Rules and Rules for Buildings. European Committee for Standardization.

Fiore, A., Monaco, P., 2009. Pod-based representation of the alongwind equivalent static force for long-span bridges. Wind and Structures, An International Journal 12, 239-257.

Ho, T.C.E., Surry, D., Morrish, D., Kopp, G.A., 2005. The uwo contribution to the nist aerodynamic database for wind loads on low buildings: Part 1. archiving format and basic aerodynamic data. Journal of Wind Engineering and Industrial Aerodynamics 93, 1-30.

Holmes, J.D., 1988. Distribution of peak wind loads on a low-rise building. Journal Of Wind Engineering and Industrial Aerodynamics 29, 59-67.

Kareem, A., Zhao, J., 1994. Analysis of non-gaussian surge response of tension leg platforms under wind loads. Journal of Offshore Mechanics and Arctic Engineering 116, 137-144.

Kasperski, M., 1992. Extreme wind load distributions for linear and nonlinear design. Engineering Structures 14, 27-34.

Katsumura, A., Tamura, Y., Nakamura, O., 2007. Universal wind load distribution simultaneously reproducing largest load effects in all subject members on large-span cantilevered roof. Journal of Wind Engineering and Industrial Aerodynamics 95, 1145-1165.

Kwon, D.K., Kareem, A., 2011. Peak factors for non-gaussian load effects revisited. Journal of Structural Engineering 137, 1611-1619.

Main, J., 2006. University of western ontario data sets. 
Main, J.A., Fritz, W.P., 2006. Database-assisted design for wind: concepts, software, and examples for rigid and flexible buildings. National Institute of Standards and Technology, Technology Administration, US Department of Commerce.

Papoulis, A., 1965. Probability, Random Variables, and Stochastic Processes. McGraw Hill, New York.

Peng, X., Yang, L., Gavanski, E., Gurley, K., Prevatt, D., 2014. A comparison of methods to estimate peak wind loads on buildings. Journal of Wind Engineering and Industrial Aerodynamics 126, $11-23$.

Repetto, M.P., Solari, G., 2004. Equivalent static wind actions on vertical structures. Journal of Wind Engineering and Industrial Aerodynamics 92, 335-357.

Tamura, Y., Fujii, K., Ueda, H., 1992. Design wind loads for beams supporting flat roofs. Journal of Wind Engineering and Industrial Aerodynamics 43, 1841-1852.

Tamura, Y., Kikuchi, H., Hibi, K., 2001. Extreme wind pressure distributions on low-rise building models. Journal of Wind Engineering and Industrial Aerodynamics 89, 1635-1646.

Tamura, Y., Kikuchi, H., Hibi, K., 2002. Actual extreme pressure distributions and lrc formula. Journal of Wind Engineering and Industrial Aerodynamics 90, 1959-1971.

Winterstein, S.R., 1988. Nonlinear vibration models for extremes and fatigue. Journal of Engineering Mechanics 114, 1772-1790.

Zhou, X., Gu, M., Li, G., 2011. Application research of constrained least-squares method in computing equivalent static wind loads, in: Proceedings of the 13th International Conference on Wind Engineering. 